\title{
Multiscale analysis using a coupled discrete/finite element model
}

\author{
Jerzy Rojek ${ }^{\dagger}$ \\ Institute of Fundamental Technological Research, Swietokrzyska 21, 00-049 Warsaw, Poland \\ Eugenio Oñate \\ International Center for Numerical Methods in Engineering (CIMNE) \\ Universidad Politécnica de Cataluña, Campus Norte UPC, 08034 Barcelona, Spain
}

(Received July 31, 2007, Accepted November 11, 2007)

\begin{abstract}
The present paper presents multiscale modelling via coupling of the discrete and finite element methods. Theoretical formulation of the discrete element method using spherical or cylindrical particles has been briefly reviewed. Basic equations of the finite element method using the explicit time integration have been given. The micr-macro transition for the discrete element method has been discussed. Theoretical formulations for macroscopic stress and strain tensors have been given. Determination of macroscopic constitutive properties using dimensionless micro-macro relationships has been proposed. The formulation of the multiscale DEM/FEM model employing the DEM and FEM in different subdomains of the same body has been presented. The coupling allows the use of partially overlapping DEM and FEM subdomains. The overlap zone in the two coupling algorithms is introduced in order to provide a smooth transition from one discretization method to the other. Coupling between the DEM and FEM subdomains is provided by additional kinematic constraints imposed by means of either the Lagrange multipliers or penalty function method. The coupled DEM/FEM formulation has been implemented in the authors' own numerical program. Good performance of the numerical algorithms has been demonstrated in a number of examples.
\end{abstract}

Keywords: multiscale modelling; discrete element method; finite element method; coupling

\section{Introduction}

The discrete element method (DEM) and finite element method (FEM) are two different numerical methods based on two different approaches to material modelling. The finite element method is a discretization method in continuum problems and the equations of the discrete element method are obtained directly from the mathematical description of a discrete material. A discrete element model has all the features of a model on the micro- or meso-scale, while the finite element method employs macroscopic models based on the continuum theory.

Integration of continuous and discrete modelling is a subject of intensive research work recently.

$\uparrow$ Corresponding Author, E-mail: jrojek@ippt.gov.pl

†E-mail: onate@cimne.upc.edu 
One of the reasons of growing interest is possibility of multi-scale modelling of complex physical phenomena (Xiao and Belytschko 2004). Coupling the finite/discrete element methods allows us to create multiscale models where material models at different levels are employed in different subdomains of the same body. In some parts discrete model can be used, while in other parts finite element method based on the continuum mechanics model can be used. The DEM is a suitable method to model materials with discontinuities and material failure characterized with fracture (Cook and Jensen 2002). The FEM is usually a method of choice in problems involving linear and nonlinear continuous material behaviour. In the proposed approach the DEM and FEM are treated as complementary methods. Combining different methods in one model will allow us to take advantages of each method.

Coupling of discrete and finite element methods can be achieved in different way, (Muniiza 2004, Potyondy and Cundall 2004, Young et al. 2004). The unified discrete/finite element formulation presented in Rojek and Oñate (2004) and Oñate and Rojek (2004) enabled simultaneous use of the discrete element and finite element methods in disjoint parts of the model interacting with each other by means of contact forces. The present paper presents extension of the coupled discrete/finite element formulation developed in Rojek (2007) allowing us to use the two coupled methods in different subdomains of the same body.
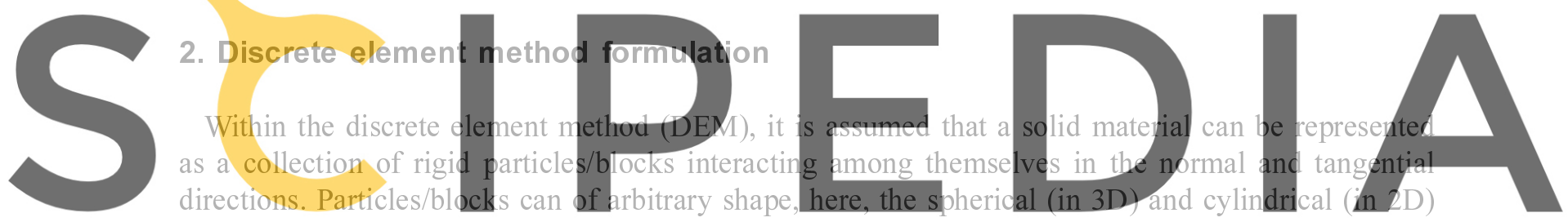

particles are employed. Discrete element formulation using spherical or cylindrical particles was

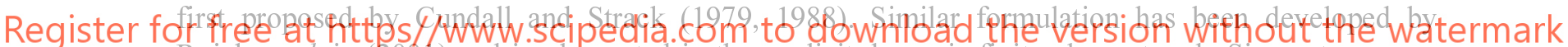
Rojek et al. in (2001) and implemented in the explicit dynamic finite element code Simpact.

The translational and rotational motion of rigid spherical or cylindrical elements (particles) is governed by the standard equations of rigid body dynamics. For the $i$-th element (Fig. 1) we have

$$
m_{i} \ddot{\boldsymbol{u}}_{i}=\boldsymbol{F}_{i}
$$

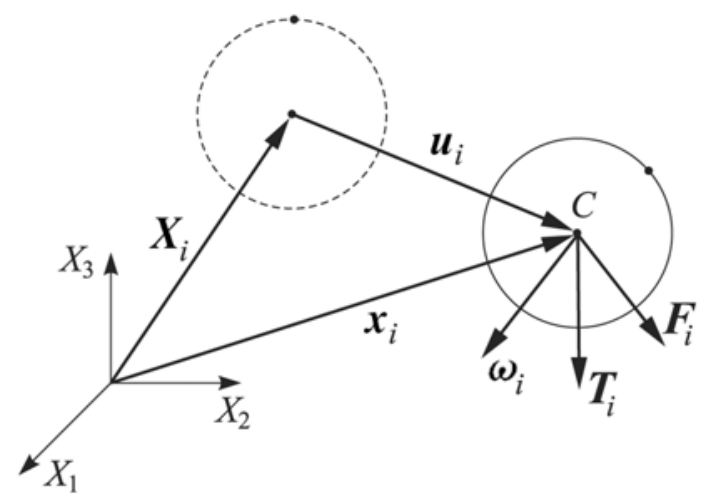

Fig. 1 Motion of a discrete element 


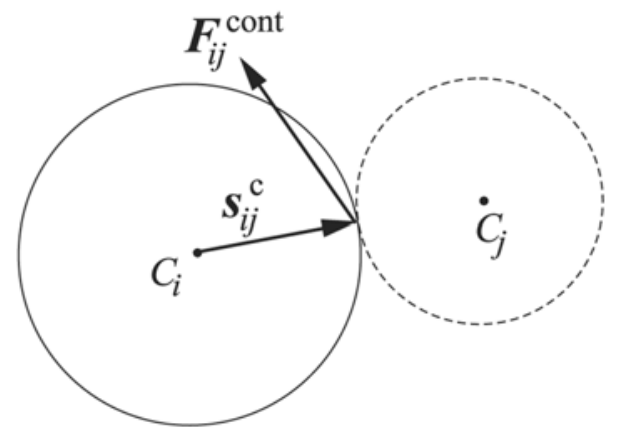

Fig. 2 Contact interaction between two discrete elements

$$
J_{i} \dot{\omega}_{i}=T_{i}
$$

where $\boldsymbol{u}_{i}$ is the element centroid displacement in a fixed (inertial) coordinate frame $X, \omega_{i}$ - the angular velocity, $m_{i}$ - the element mass, $J_{i}$ - the moment of inertia, $\boldsymbol{F}_{i}$ - the resultant force, and $\boldsymbol{T}_{i}$ the resultant moment about the central axes. The form of the rotational Eq. (2) is valid for spheres and cylinders (in 2D) and is simplified with respect to a general form for an arbitrary rigid body with the rotational inertial properties represented by a second order tensor. Vectors $F_{i}$ and $T_{i}$ are

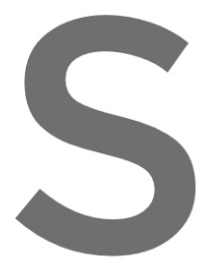
Sums of: (i) all forces and moments applied to the
respectively, (ii) contact interactions with neighb
the number of elements being in contact with the
resulting from external damping, $\boldsymbol{F}_{i}^{\text {damp }}$ and $T_{i}^{\text {damp }}$
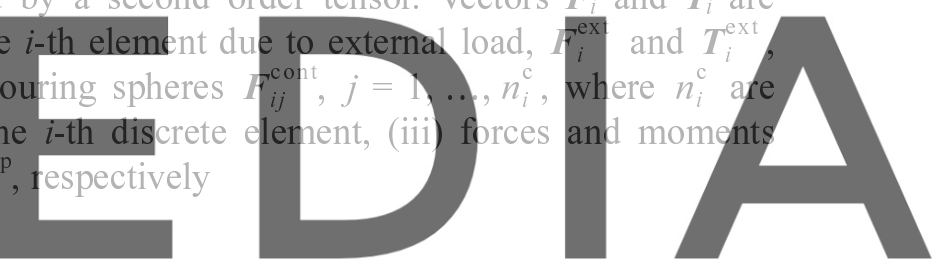

$$
\boldsymbol{F}_{i}=\boldsymbol{F}_{i}^{\mathrm{ext}}+\sum_{i j}^{n_{i}} \boldsymbol{F}_{i j}^{\mathrm{cont}}+\boldsymbol{F}_{i}^{\mathrm{damp}}=\boldsymbol{F}_{i}^{\mathrm{ext}}+\boldsymbol{F}^{\mathrm{cont}}+\boldsymbol{F}_{i}^{\mathrm{damp}}
$$

(3)

Register for free at https//wWw.scipedila.com to download the version without the watermark

$$
T_{i}=T_{i}^{\mathrm{ext}}+\sum_{j=1}^{n_{i}^{\mathrm{c}}} \mathbb{S}_{i j}^{\mathrm{c}} \times \mathbb{F}_{i j}^{\mathrm{cont}}+T_{i}^{\mathrm{damp}}=T_{i}^{\mathrm{ext}}+\mathbb{F}_{i}^{\mathrm{cont}}+T_{i}^{\mathrm{damp}}
$$

where $\boldsymbol{S}_{i j}^{\mathrm{c}}$ is the vector connecting the centre of mass of the $i$-th element with the contact point with the $j$-th element (Fig. 2).

Eqs. of motion (1) and (2) for a set of $N$ discrete elements can be written in a matrix form as follows:

where:

$$
\begin{aligned}
& \mathbf{M}_{\mathrm{D}} \ddot{\mathbf{r}}_{\mathrm{D}}=\mathbf{F}_{\mathrm{D}} \\
& \mathbf{J}_{\mathrm{D}} \dot{\boldsymbol{\Omega}}_{\mathrm{D}}=\mathbf{T}_{\mathrm{D}}
\end{aligned}
$$

$$
\begin{gathered}
\mathbf{M}_{\mathrm{D}}=\left[\begin{array}{ccc}
m_{1} \mathbf{1}_{3 \times 3} & & \mathbf{0} \\
& \ddots & \\
\mathbf{0} & m_{N} \mathbf{1}_{3 \times 3}
\end{array}\right], \mathbf{J}_{\mathrm{D}}=\left[\begin{array}{ccc}
J_{1} \mathbf{1}_{3 \times 3} & & \mathbf{0} \\
& \ddots & \\
\mathbf{0} & & J_{N} \mathbf{1}_{3 \times 3}
\end{array}\right] \\
\mathbf{r}_{\mathrm{D}}=\left\{\boldsymbol{u}_{1}, \ldots, \boldsymbol{u}_{N}\right\}^{\mathrm{T}}, \quad \boldsymbol{\Omega}_{\mathrm{D}}=\left\{\boldsymbol{\omega}_{1}, \ldots, \boldsymbol{\omega}_{N}\right\}^{\mathrm{T}}
\end{gathered}
$$




$$
\mathbf{F}_{\mathrm{D}}=\left\{\boldsymbol{F}_{1}, \ldots, \boldsymbol{F}_{N}\right\}^{\mathrm{T}}, \quad \mathbf{T}_{\mathrm{D}}=\left\{\boldsymbol{T}_{1}, \ldots, \boldsymbol{T}_{N}\right\}^{\mathrm{T}}
$$

Eqs. of motion (5) and (6) are integrated in time using the central difference scheme. The time integration operator for the translational motion at the $n$-th time step is as follows:

$$
\begin{gathered}
\ddot{\mathbf{r}}_{\mathrm{D}}^{n}=\mathbf{M}_{\mathrm{D}}^{-1} \mathbf{F}_{\mathrm{D}}^{n} \\
\dot{\mathbf{r}}_{\mathrm{D}}^{n+1 / 2}=\dot{\mathbf{r}}_{\mathrm{D}}^{n-1 / 2}+\ddot{\mathbf{r}}_{\mathrm{D}}^{n} \Delta t \\
\mathbf{r}_{\mathrm{D}}^{n+1}=\mathbf{r}_{\mathrm{D}}^{n}+\dot{\mathbf{r}}_{\mathrm{D}}^{n+1 / 2} \Delta t
\end{gathered}
$$

The first two steps in the integration scheme for the rotational motion are identical to those given by Eqs. (10) and (11):

$$
\begin{gathered}
\dot{\Omega}_{\mathrm{D}}^{n}=\mathbf{J}_{\mathrm{D}}^{-1} \mathbf{T}_{\mathrm{D}}^{n} \\
\Omega_{\mathrm{D}}^{n+1 / 2}=\Omega_{\mathrm{D}}^{n-1 / 2}+\dot{\Omega}_{\mathrm{D}}^{n} \Delta t
\end{gathered}
$$

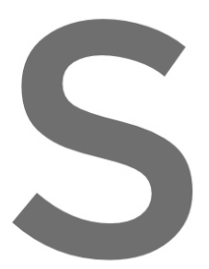

The vector of incremental
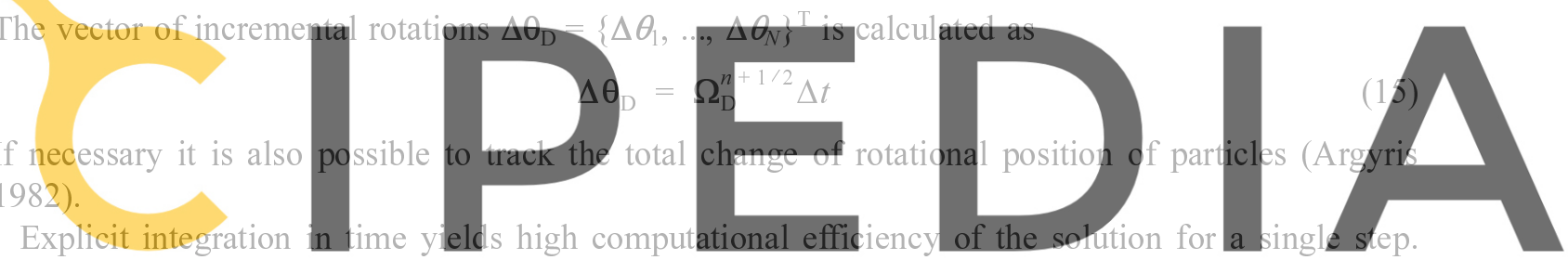

The disadvantage of the explicit integration scheme is its conditional numerical stability imposing

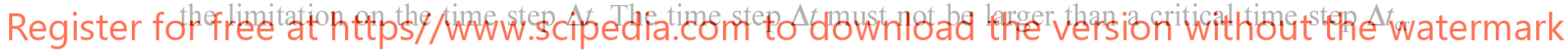

$$
\Delta t \leq \Delta t_{\mathrm{cr}}
$$

determined by the highest natural frequency of the system $v_{\max }$

$$
\Delta t_{\mathrm{cr}}=\frac{2}{v_{\max }}
$$

Exact determination of the highest frequency $v_{\max }$ would require solution of the eigenvalue problem defined for the whole system of connected rigid particles. The maximum frequency of the whole system can be estimated as the maximum of natural frequencies $v_{i}^{e}$ of subsets of connected particles surrounding each particle $e$, cf. (Belytscho et al. 1985):

$$
v_{\max } \leq v_{\max }^{D}, \quad \text { where } \quad v_{\max }^{D}=\max _{i, e} v_{i}^{e}
$$

The contact force between two elements ${ }^{1} \boldsymbol{F}^{\text {cont }}$ can be decomposed into normal and tangential components, $\boldsymbol{F}_{\mathrm{n}}{ }^{\text {cont }}$ and $\boldsymbol{F}_{\mathrm{t}}{ }^{\text {cont }}$, respectively

$$
\boldsymbol{F}^{\text {cont }}=\boldsymbol{F}_{\mathrm{n}}^{\text {cont }}+\boldsymbol{F}_{\mathrm{t}}^{\text {cont }}=F_{\mathrm{n}}^{\text {cont }} \boldsymbol{n}+\boldsymbol{F}_{\mathrm{t}}^{\text {cont }}
$$

${ }^{1}$ In the next part of this section indices denoting the elements will be omitted. 


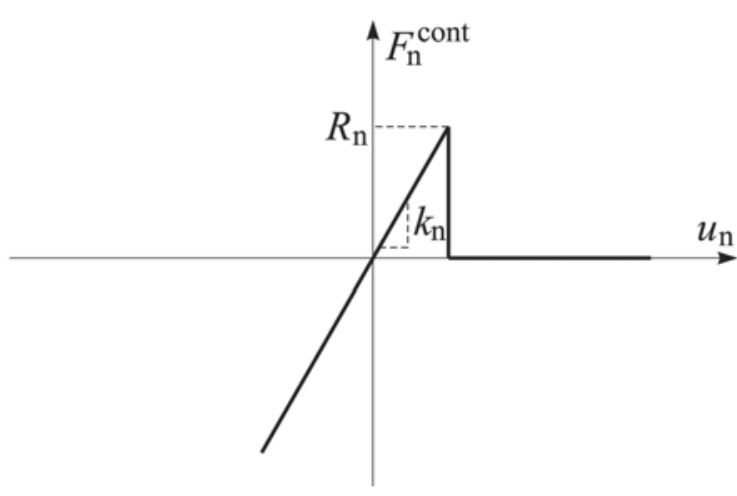

a)

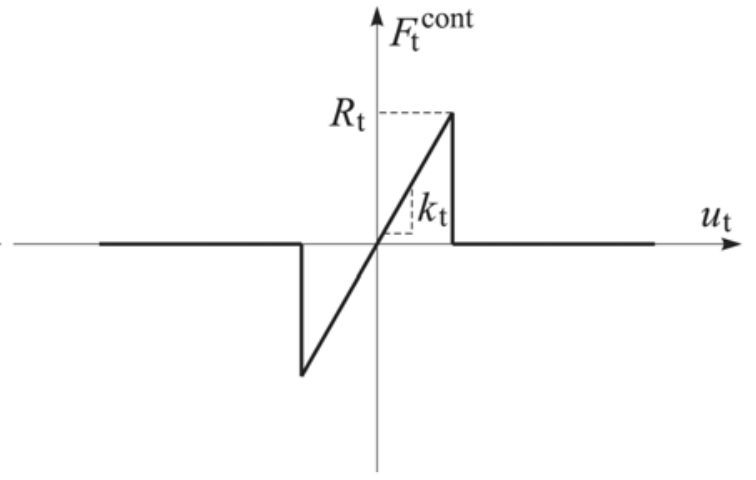

b)

Fig. 3 Force-displacement relationships for the elastic perfectly brittle model: a) in the normal direction, b) in the tangential direction

where $\boldsymbol{n}$ is the unit vector normal to the particle surface at the contact point.

The contact forces $F_{\mathrm{n}}^{\text {cont }}$ and $\boldsymbol{F}_{\mathrm{t}}^{\text {cont }}$ are obtained using a constitutive model formulated for the contact between two rigid spheres. In the present formulation rock materials are modelled using elastic perfectly brittle neighbouring particles. propagation of material perfectly brittle model ar both normal and tange
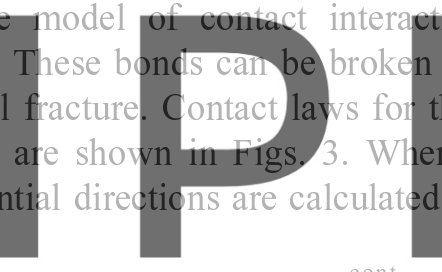

$$
F_{\mathrm{n}}^{\mathrm{cont}}=k_{\mathrm{n}} u_{\mathrm{n}}
$$
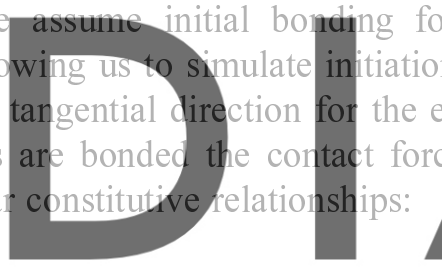

$(20)$

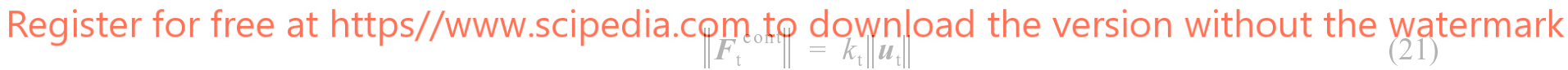

where: $F_{\mathrm{n}}{ }^{\text {cont }}$-normal contact force, Fcont $\boldsymbol{F}_{\mathrm{t}}{ }^{\text {cont }}$-tangential contact force, $k_{\mathrm{n}}$-interface stiffness in the normal direction, $k_{\mathrm{t}^{-}}$interface stiffness in the tangential direction, $u_{\mathrm{n}}$-normal relative displacement, $\boldsymbol{u}_{\mathrm{t}}$-tangential relative displacement.

Cohesive bonds are broken instantaneously when the interface strength is exceeded in the tangential direction by the tangential contact force or in the normal direction by the tensile contact force. The failure (decohesion) criterion can be written as:

$$
\begin{gathered}
F_{\mathrm{n}}^{\text {cont }} \leq R_{\mathrm{n}} \\
\left\|\boldsymbol{F}_{\mathrm{t}}^{\text {cont }}\right\| \leq R_{\mathrm{t}}
\end{gathered}
$$

where: $R_{\mathrm{n}}$-interface strength in the normal direction, $R_{\mathrm{t}}$-interface strength in the tangential direction.

In the absence of cohesion the normal contact force can be compressive only $\left(R_{n} \leq 0\right)$ and tangential contact force can be nonzero due to friction

$$
\left\|\boldsymbol{F}_{\mathrm{t}}^{\mathrm{cont}}\right\|=\mu\left|F_{\mathrm{n}}^{\mathrm{cont}}\right|
$$

if $R_{\mathrm{n}}<0$ or zero otherwise. The friction force is given by Eq. (24) expressing the Coulomb friction law, with $\mu$ being the Coulomb friction coefficient. 
A quasi-static state of equilibrium of the assembly of particles can be achieved by application of adequate damping. Damping is necessary to dissipate kinetic energy. Damping terms $\boldsymbol{F}_{i}^{\text {damp }}$ and $\boldsymbol{T}_{i}^{\text {damp }}$ in Eqs. (3) and (4) in the present work are of non-viscous type and are given by:

$$
\begin{gathered}
\boldsymbol{F}_{i}^{\text {damp }}=-\alpha^{t} \| \boldsymbol{F}_{i}^{\text {ext }}+\boldsymbol{F}_{i}^{\text {cont } \|} \frac{\dot{\boldsymbol{u}}_{i}}{\left\|\dot{\boldsymbol{u}}_{i}\right\|} \\
\boldsymbol{T}_{i}^{\text {damp }}=-\alpha^{r}\left\|\boldsymbol{T}_{i}\right\| \frac{\boldsymbol{\omega}_{i}}{\left\|\boldsymbol{\omega}_{i}\right\|}
\end{gathered}
$$

where $\alpha^{t}$ and $\alpha^{r}$, are respective damping constants for translational and rotational motion.

\section{Finite element method formulation}

In the present work the so-called explicit dynamic formulation of the finite element method is used. The explicit FEM is based on the solution of discretized equations of motion written in the current configuration in the following form:
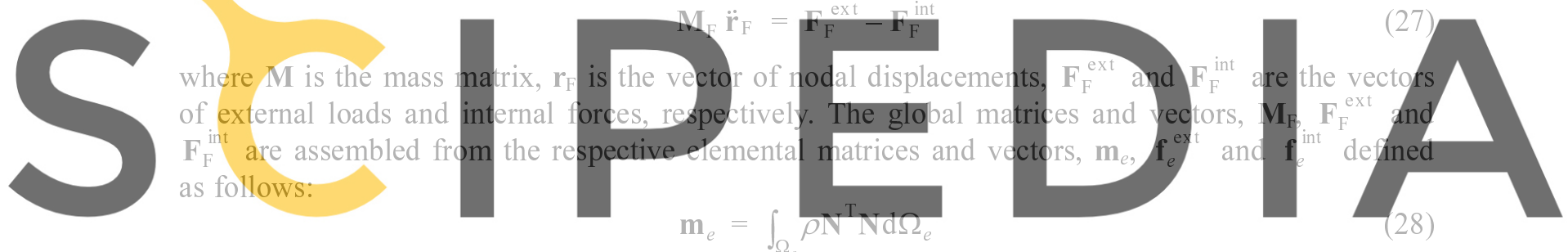

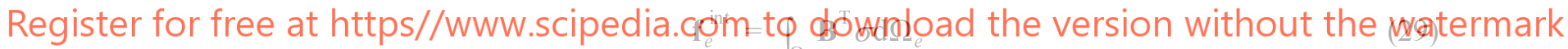

$$
\mathbf{f}_{e}^{\text {ext }}=\int_{\Omega_{e}} \mathbb{N}^{\mathrm{T}} \rho \mathbf{b d} \Omega_{e}+\int_{\Gamma_{e}} \mathbf{N}^{\mathrm{T}} \mathbf{t} \mathrm{d} \Gamma_{e}
$$

where $\rho$ is the mass density, $\sigma$ is the Cauchy stress tensor, $\mathbf{b}$ are the body forces, $\mathbf{t}$ is the surface traction, $\mathbf{N}$ is thematrix of interpolation (shape) functions and $\mathbf{B}$ is the linear strain-displacement operator matrix.

Similarly to the DEM algorithm, the central difference scheme is used for time integration of Eq. (27):

$$
\begin{gathered}
\ddot{\mathbf{r}}_{\mathrm{F}}^{n}=\mathbf{M}_{\mathrm{F}}^{-1}\left(\mathbf{F}_{\mathrm{F}}^{\text {ext }}-\mathbf{F}_{\mathrm{F}}^{\text {int }}\right)^{n} \\
\dot{\mathbf{r}}_{\mathrm{F}}^{n+1 / 2}=\dot{\mathbf{r}}_{\mathrm{F}}^{n-1 / 2}+\ddot{\mathbf{r}}_{\mathrm{F}}^{n} \Delta t \\
\mathbf{r}_{\mathrm{F}}^{n+1}=\mathbf{r}_{\mathrm{F}}^{n}+\dot{\mathbf{r}}_{\mathrm{F}}^{n+1 / 2} \Delta t
\end{gathered}
$$

Use of a diagonalized mass matrix in Eq. (27) allows us to decouple the set of equations, and eliminates the necessity of matrix inversion in Eq. (31). This leads to a very efficient solution for a single step of time integration. 

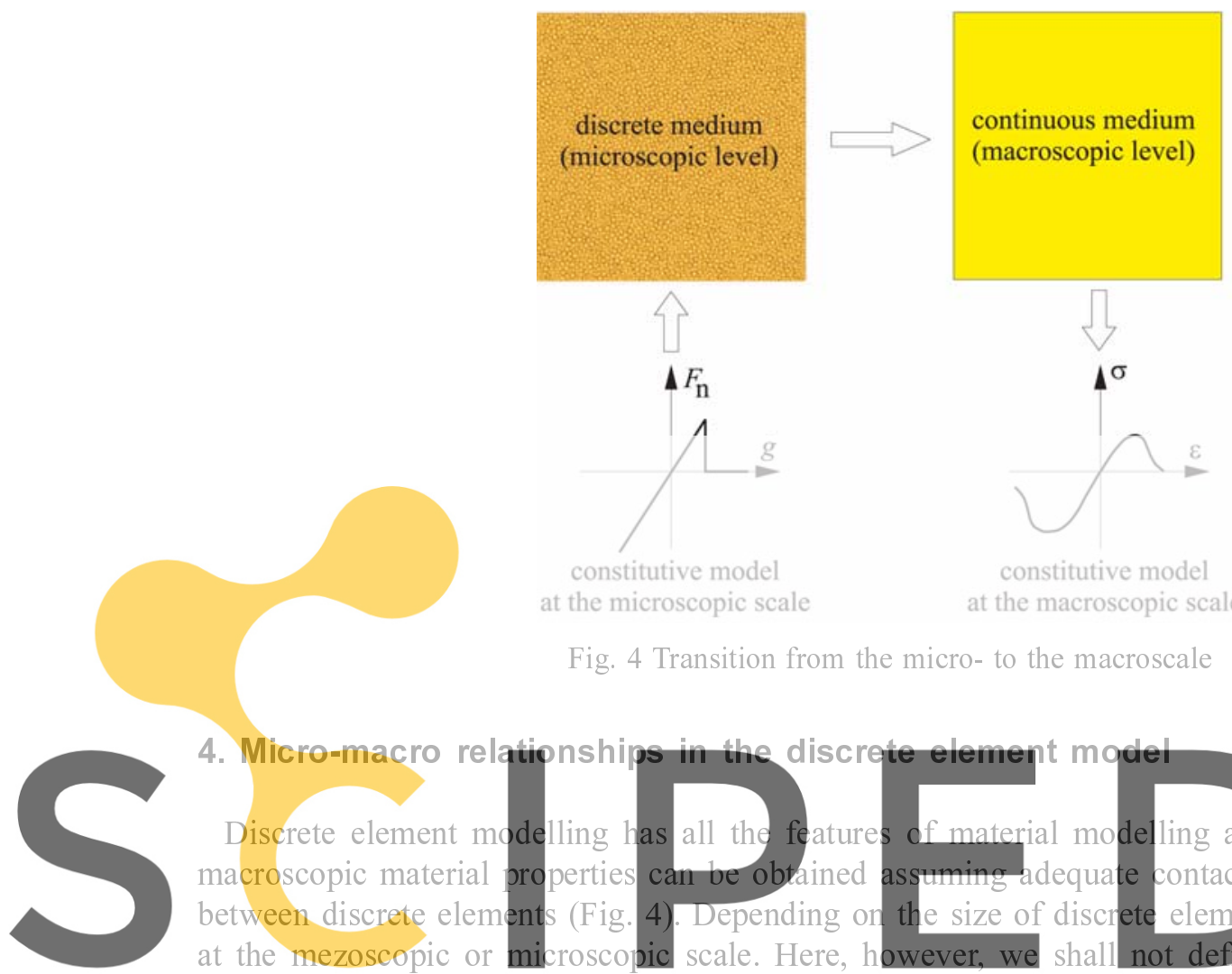

4. Micro-macro relationships in the discrete element Discrete element modelling has all the features

macroscopic material pro

between discrete elements

at the mezoscopic or

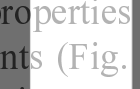

croscopic
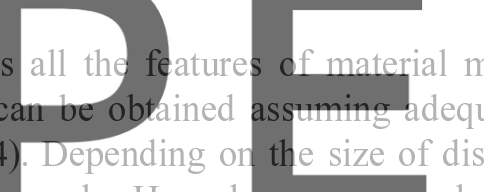

elements, referring to the models as microscopic models.

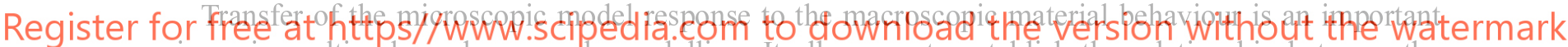
issue in multiscale or lower scale modelling. It allows us to establish the relationship between the microscopic model parameters and macroscopic variables. Transition from the microscopic to macroscopic level description allows verification of the microscopic model by comparison of the calculated macroscopic parameters with those measured in a laboratory.

Effective macroscopic variables and properties in micromechanical models can be determined by various analytical and numerical homogenization and averaging methods (Miehe et al. 2002, Ramm et al. 2003, Kouznetsova 2002, Lätzel 2003). In this work averaging methods based on the concept of the representative volume element (RVE) will be used.

\subsection{Problem formulation}

We shall consider a set of $n_{d e}$ spherical or cylindrical discrete elements $D=\left\{d_{i}\right\}, i=1, n_{d e}$ occupying the domain

$$
\tilde{\Omega}_{\mathrm{D}}=\sum_{i=1}^{i=n_{d e}} d_{i} \subset \Omega_{\mathrm{D}}
$$

in the Euclidean space $E^{n_{s d}}$, where $n_{s d}=2$ or 3 (Fig. 5). We assume that the discrete elements interact among themselves with contact forces determined according the model described in Sec. 2, 


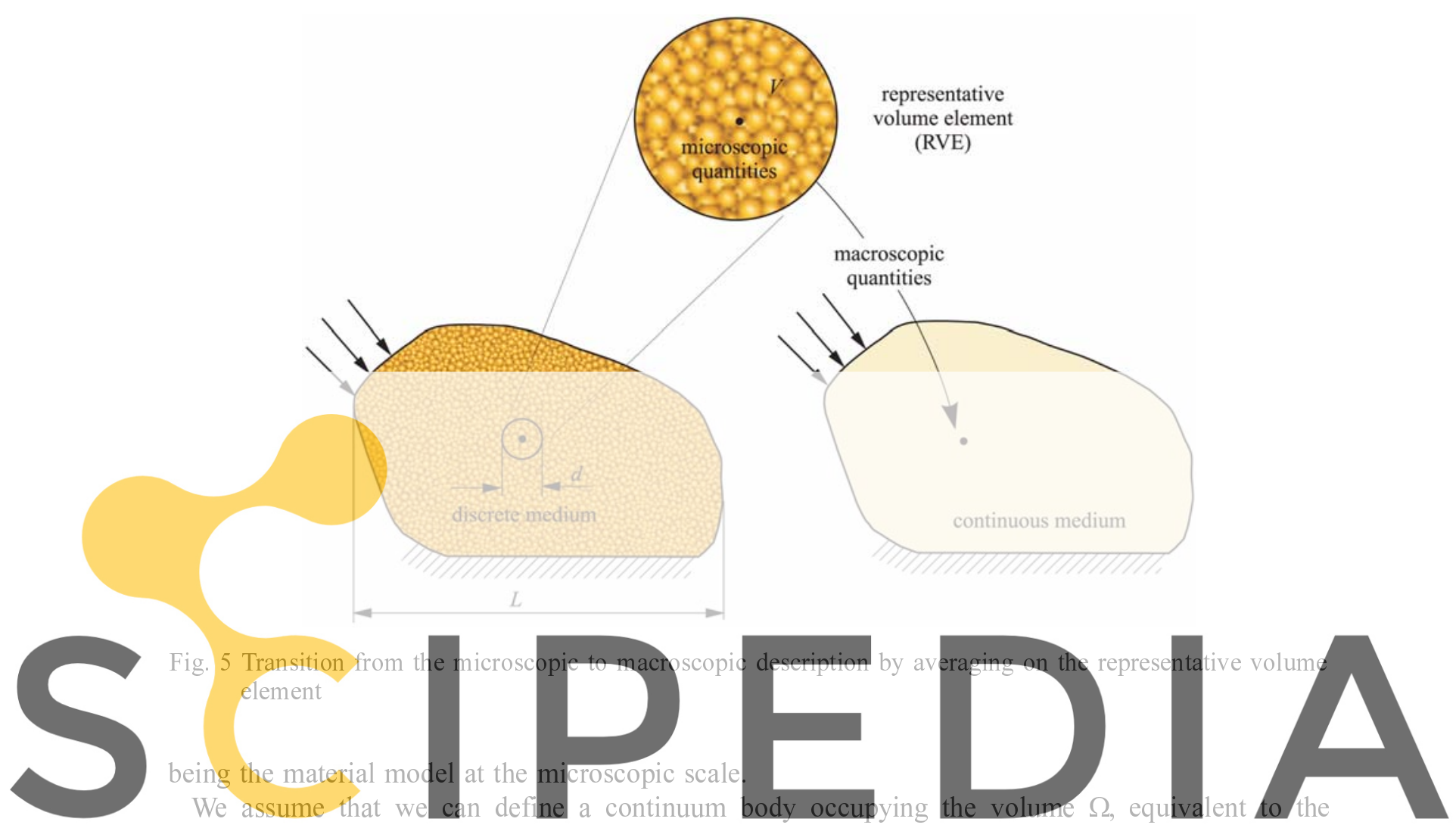

discrete medium considered. The body occupying the volume $\Omega$ can be regarded continuous with

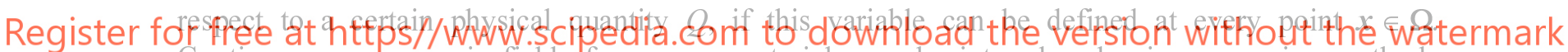
Continuous macroscopic fields for porous materials can be intro duced using averaging methods

based on the concept of the representative volume element (RVE) (Christensen 1979, Nemat-Nasser

and Hori 1993). The idea of this approach is shown schematically in Fig. 5. Around each point $\boldsymbol{x} \in \boldsymbol{\Omega}$ we define a representative element having volume $V$, over which the quantity $Q$ will be averaged according to the following formula:

$$
\bar{Q}=\langle Q\rangle=\frac{1}{V} \int_{V} Q \mathrm{~d} V
$$

The average $\langle Q>$, obtained in this way, will be assigned to the considered point $\boldsymbol{x}$. Performing the averaging procedure for all points in the domain $\Omega$, we transform the microscopic discrete description into a macroscopic continuum description.

The size of the representative volume element should be much smaller than macroscopic dimensions of the considered body $\Omega(d<<L)$. The RVE size, however, should be sufficiently large in order to eliminate fluctuations typical for the lower scale (Fig. 6). On the other hand the RVE should not be too large, if we want to treat the values of averaged quantities as local ones (if the field of averaged quantity is non-homogeneous).

The RVE shape can be arbitrary-Eq. (35) is valid for any shape. In mathematical homogenization methods requiring a solution of the boundary problem, a square (in 2D) or cubic (in 3D) RVE is usually assumed because it is easy to define appropriate boundary conditions for these shapes. In 


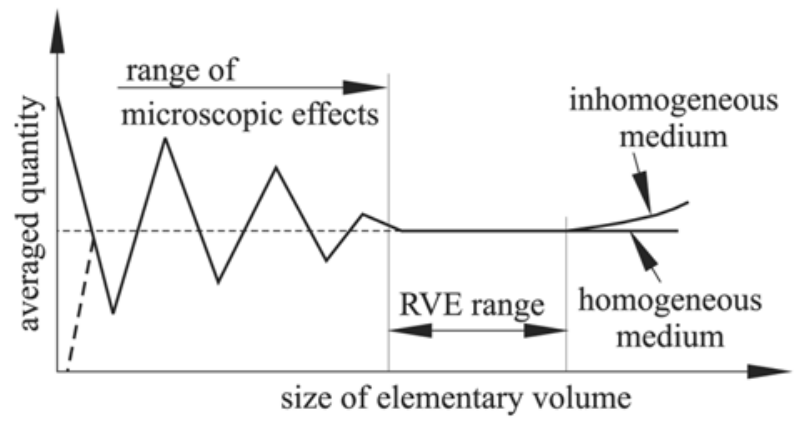

Fig. 6 Determination of the size of the representative volume element

our case circular (in 2D) or spherical (in 3D) representative volume elements centred at the points $\boldsymbol{x} \in \Omega$ will be used. Special treatment will be applied in case of points on the boundary or near the boundary. In such cases the averaging will be performed considering the intersection of the RVE with the volume $\Omega$ occupied by the body.
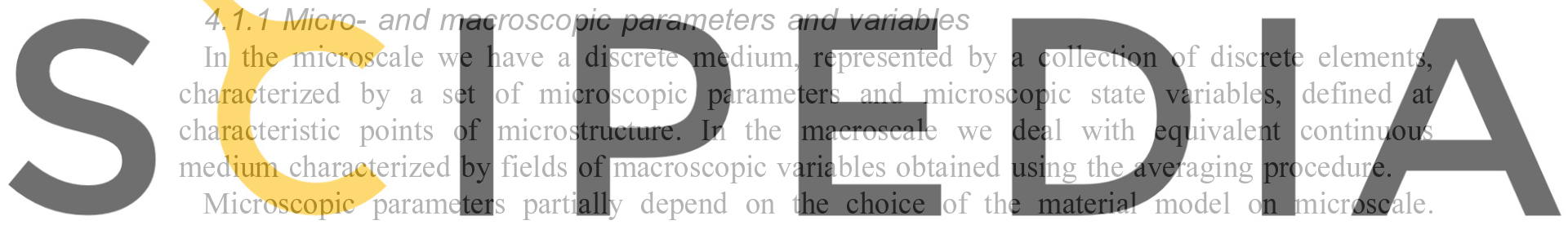

Assuming the elastic-brittle model of interaction between discrete elements described in Sec. 2 we

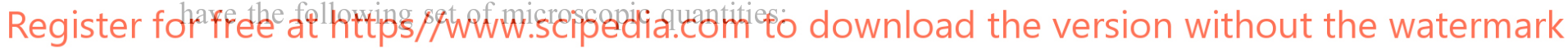

- geometrical and physical parameters:

$r$-radius, parameter defining the size of discrete elements

$\rho$ - mass density

$n$ - porosity, characterizing compaction

- constitutive parameters:

$k_{\mathrm{n}}$ - contact stiffness in the normal direction

$k_{\mathrm{s}}$ - contact stiffness in the tangential direction

$R_{\mathrm{n}}$ - interface strength in the normal direction

$R_{\mathrm{S}}$ - interface strength in the tangential direction

$\mu$ - Coulomb friction coefficient

$\alpha^{t}$ - damping coefficient for translational motion

$\alpha^{r}$ - damping coefficient for rotational motion

- state variables:

$g$ - relative displacement in the normal direction

$\boldsymbol{u}_{\mathrm{s}}$ - relative displacement in the tangential direction

$F_{\mathrm{n}}$ - normal interaction force

$\boldsymbol{F}_{\mathrm{s}}$ - tangential interaction force, state of the cohesive bond (active or broken) 
In the description of the macroscopic state we will introduce the following quantities:

- geometrical and physical parameters:

$L$ - characteristic dimension of the considered body

$\bar{\rho}$ - averaged mass density

- constitutive parameters:

$\boldsymbol{C}$ - constitutive tensor

alternatively constitutive properties can be defined by the following parameters like, for instance,

$E$ - Young's modulus

$v$ - Poisson's ratio

$\sigma_{\mathrm{c}}$ - uniaxial compressive strength

$\sigma_{t}-$ tensile strength

- state variables:

$\varepsilon$-strain tensor

$\sigma$-stress tensor

\subsection{Averaged stress tensor}

4.2.1 Two-level averaging procedure

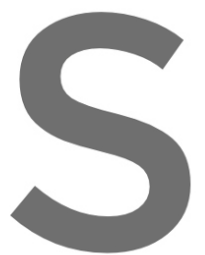

The averaging procedure to use of the concept of twe averaging over the representative Thus we obtain the qu discrete element. In th one discrete element, containing
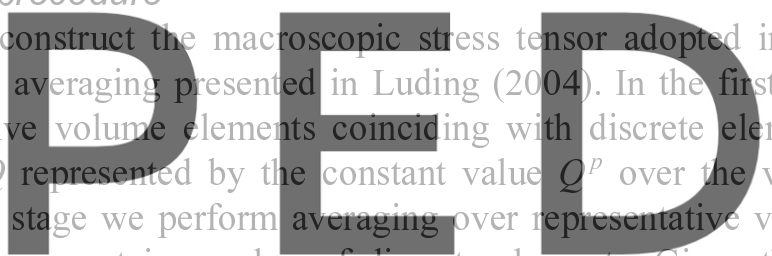

$Q^{p}$ over elements, the integral in Eq. (35) can be replaced by the following sum:

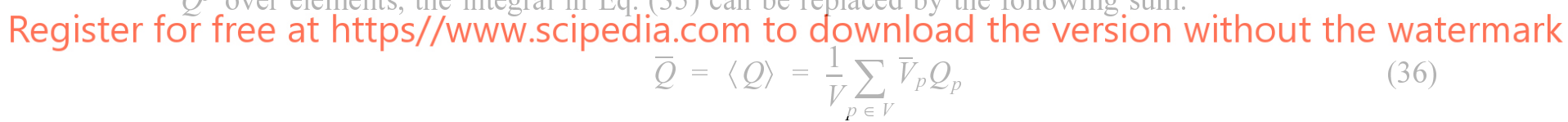

where $\bar{V}_{p}$ is the intersection of the $p$-the discrete element and the representative volume element $V$ :

$$
\bar{V}_{p}=V_{p} \cap V
$$

\subsubsection{Stress tensor for a single discrete element}

The stress tensor $\sigma_{p}$ for a single discrete element will be calculated using of Eq. (35) for the transposed tensor over the element volume $V_{p}$

$$
\sigma_{p}^{\mathrm{T}}=\frac{1}{V_{p}} \int_{V_{p}} \sigma^{\mathrm{T}} \mathrm{d} V
$$

In calculation of the stress tensor we make use of the following identity :

$$
\sigma^{\mathrm{T}}=\nabla \boldsymbol{x} \cdot \sigma^{\mathrm{T}}=\nabla \cdot(\boldsymbol{x} \sigma)-\boldsymbol{x}(\nabla \cdot \sigma)
$$

where we introduced the unit tensor $\boldsymbol{I}=\nabla \boldsymbol{x}$. Substituting identity (39) into Eq. (38) we obtain

$$
\sigma_{p}^{\mathrm{T}}=\frac{1}{V_{p}}\left(\int_{V_{p}} \nabla \cdot(\boldsymbol{x} \sigma) \mathrm{d} V-\int_{V_{p}} \boldsymbol{x}(\nabla \cdot \sigma) \mathrm{d} V\right)
$$


Assuming the static equilibrium and lack of mass loading the second integral in Eq. (40) disappears:

$$
\sigma_{p}^{\mathrm{T}}=\frac{1}{V_{p}} \int_{V_{p}} \nabla \cdot(\boldsymbol{x} \boldsymbol{\sigma}) \mathrm{d} V
$$

Employing the Gauss' law to Eq. (41), the volume integral can be transformed into the surface one:

$$
\sigma_{p}^{\mathrm{T}}=\frac{1}{V_{p}} \int_{S_{p}}(\boldsymbol{x} \boldsymbol{\sigma}) \cdot \boldsymbol{n} \mathrm{d} S
$$

where $\boldsymbol{n}$ is the unit vector normal to the element surface $S_{p}$ and directed outwards. Making use of the stress definition

$$
\sigma \cdot \boldsymbol{n}=\boldsymbol{t}
$$

with $t$ being the stress vector, Eq. (42) is rewritten in the following form:

$$
\sigma_{p}^{\mathrm{T}}=\frac{1}{V_{p}} \int_{S_{p}} \boldsymbol{x t} \mathrm{d} S
$$

Taking into account that surface loading is produced by concentrated forces $\boldsymbol{F}_{c}$ acting on the small surfaces $\delta S_{c}$, the integral in Eq. (44) can be written as the following sum:

$$
\sigma_{p}^{\mathrm{T}}=\frac{1}{V_{p_{c}}} \sum_{p=1}^{n_{p c}} \boldsymbol{x}_{c} \frac{\boldsymbol{F}^{c}}{\delta S_{c}} \delta S_{c}=\frac{1}{V_{p}} \sum_{c=1}^{n_{p c}} \boldsymbol{x}_{c} \boldsymbol{F}^{c}
$$

where $n_{p c}$ is the number of elements being in contact with the $p$-th element. Transposing Eq. (45), we obtain the expression defining the stress tensor $\sigma_{p}$ for a single particle

$$
\sigma_{p}=\frac{1}{V_{p}} \sum_{c=1}^{n_{p c}} \boldsymbol{F}^{c} \boldsymbol{x}_{c}
$$

Writing the position vector of the contact point $\boldsymbol{x}_{c}$ as the sum of the position vector of the element center, $\boldsymbol{x}_{p}$, and the branch vector, $\boldsymbol{s}_{p}^{c}$, connecting the element center with the contact point

$$
\boldsymbol{x}_{c}=\boldsymbol{x}_{p}+\boldsymbol{s}_{p}^{c}
$$

we obtain

$$
\sigma_{p}=\frac{1}{V_{p}}\left(\sum_{c=1}^{n_{p c}} \boldsymbol{F}^{c}\right) \boldsymbol{x}_{p}+\frac{1}{V_{p_{c}}} \sum_{c=1}^{n_{p c}} \boldsymbol{F}^{c} \boldsymbol{s}_{p}^{c}
$$

For the static equilibrium assumed, the first integral in Eq. (48) disappears

$$
\sum_{c=1}^{n_{p c}} \boldsymbol{F}^{c}=\mathbf{0}
$$

which finally gives the stress tensor for a single discrete element in the following form:

$$
\sigma_{p}=\frac{1}{V_{p}} \sum_{c=1}^{n_{p c}} \boldsymbol{F}^{c} \boldsymbol{s}_{p}^{c}
$$

\subsubsection{Averaged stress tensor}

After calculation of stresses for single elements $\sigma_{p}$, we will perform averaging over representative 
volume elements, defined for selected ${ }^{2}$ points $\boldsymbol{x} \in \boldsymbol{\Omega}$. Given constant stresses over elements, the averaging can be done according to Eq. (36). Substituting Eq. (50) into Eq. (36) we obtain the averaged stress tensor in the following form:

$$
\bar{\sigma}=\langle\sigma\rangle=\frac{1}{V} \sum_{p \in V} \bar{V}_{p} \frac{1}{V_{p_{c}}} \sum_{c=1}^{n_{p c}} \boldsymbol{F}^{c} \boldsymbol{s}_{p}^{c}
$$

\subsection{Macroscopic strain tensor}

Different approaches are possible to obtain macroscopic strains in the discrete element microscopic model. A number of formulations are based on the Voigt hypothesis which assumes that the strain is uniform in the granular assembly and that particle displacements are in accordance with the uniform strain (Liao et al. 1997, Latzel et al. 2000, Luding 2004). These assumptions allow us to derive the relationship between the uniform strain and relative displacements at contact points which are microscopic strain measures, cf. (Liao et al. 1997).

Alternative procedures to obtain macroscopic strain tensor employ averaging procedures (Bago 1996, Ramm et al. 2003, Bardet and Proubt 1989). In this approach the formal procedure to obtain the averaged strain tensor $\bar{\varepsilon}$ is started form the formation of an integral over the volume $V$ of the representative volume element (RVE)

$$
\bar{\varepsilon}=\langle\varepsilon\rangle=\frac{1}{V} \int_{V} \varepsilon \mathrm{d} V=\frac{1}{V} \int_{V} \frac{1}{2}(\nabla \boldsymbol{u}+\boldsymbol{u} \nabla) \mathrm{d} V
$$

Employing the Gauss' law the volume integral in Eq. (52) can be replaced by the surface integral

$$
\bar{\varepsilon}=\frac{1}{V} \int_{V} \frac{1}{2}(\nabla \boldsymbol{u}+\boldsymbol{u} \nabla) \mathrm{d} V=\frac{1}{V} \int_{V} \frac{1}{2}(\boldsymbol{u n}+\boldsymbol{n} \boldsymbol{u}) \mathrm{d} S
$$

where $\boldsymbol{n}$ is the unit vector normal to the boundary surface of the RVE and directed outwards. The surface integral in Eq. (53) can be calculated approximately (Bardet and Proubt 1989):

$$
\bar{\varepsilon}=\frac{1}{V} \int_{S} \frac{1}{2}(\boldsymbol{u n}+\boldsymbol{n u}) \mathrm{d} S=\frac{1}{2 V} \sum_{k=1}^{n_{s}}\left(\boldsymbol{u}_{k} \boldsymbol{n}_{k}+\boldsymbol{u}_{k} \boldsymbol{n}_{k}\right) S_{k}
$$

where $n_{s}$ is the number of elements intersecting the boundary surface, $S_{k}$ is the part of the boundary assigned to the $k$-th element, $\boldsymbol{n}_{k}$ is the unit normal vector, and $\boldsymbol{u}_{k}$ is the displacement vector of the $k$ th element. Thus the macroscopic strain can be obtained as a function of displacements of the particles on the boundary of the RVE. The relationship between the averaged (macroscopic) strain and contact relative displacements (microscopic strain measures) is implicit. The explicit relationship has been obtained by Bagi in (1996) by introducing the subdivision of the averaging domain into special cells and using this subdivision in calculation of the integral Eq. (54).

In practical applications in this work we deal with large relative displacements of discrete elements after material fracture and failure. In this case the averaged total strain would not be very meaningful, therefore the described algorithm has not been implemented in our numerical code.

\footnotetext{
${ }^{2}$ For practical reasons averaging will be carried out for selected points only, and from these points stress field can be constructed by interpolation.
} 


\subsection{Macroscopic constitutive properties}

\subsubsection{Macroscopic constitutive relationship}

Macroscopic constitutive relationship between the averaged stress tensor $\bar{\sigma}$ and the macroscopic strain tensor

$$
\bar{\sigma}=\overline{\boldsymbol{C}}: \bar{\varepsilon}
$$

defines the densor of effective constitutive properties $\overline{\boldsymbol{C}}$. Macroscopic properties for the assembly of discrete elements in the linear elastic range can be estimated theoreatically in terms of parameters defining the microscopic models (Liao and Chan 1987, Luding 2004). Determination of constitutive properties in the nonlinear range requires the use of simulation methods. In Miehe (1996) the tangent constitutive tensor $\mathbf{C}$ has been determined using numerical results and incremental form of Eq. (55):

$$
\Delta \bar{\sigma}=\overline{\boldsymbol{C}}: \Delta \overline{\boldsymbol{\varepsilon}}
$$

In this work macroscopic properties will be determined using the methodology proposed in Huang (1999) combining numerical simulation and dimensionless relationships obtained on the basis of the Buckingham $\pi$ theorem (Langhaar 1951).

\subsubsection{Dimensionless micro-macro relationships}

The Buckingham $\pi$ theorem known from dimensional analysis (Langhaar 1951) states that any physically meaningful functional relationship of $N$ variables $\Psi\left(Q_{1}, Q_{2}, \ldots, Q_{N}\right)$ can be expressed equivalently by a function of $N-r$ demensionless parameters $\Phi\left(\pi_{1}, \pi_{2}, \ldots, \pi_{N-r}\right)$, where $r$ is the number of primary dimensions (minimum independent dimensions required to specify the dimensions of all the relevant parameters), and $\mathrm{N}-r$ is the maximum number of independent parameters.

Here we will search functions defining the macroscopic material parameters: Young's modulus $E$, Poisson's ratio $\mathrm{n}$ and uniaxial compressive strength sc in terms of microscopic parameters defined in subsection 4.1.1: $r, \rho, n, k_{\mathrm{n}}, k_{\mathrm{s}}, R_{\mathrm{n}}, R_{\mathrm{s}}, \mu, \alpha^{t}, \alpha^{r}$. Macroscopic properties can also depend on other parameters, like geometrical parameters (possible scale effect) or loading velocity. Thus, the set of parameters influencing macroscopic properties will be completed with prescribed velocity on the boundary $v$ and characteristic macroscopic dimension $L$. The number of relevant parameters $N$ is 12 . We have three primary dimensions involved: mass, length, time $(r=3)$.

On the basis of the Buckingham $\mathrm{p}$ theorem we can assume there are 9 independent parameters: $\left\{k_{\mathrm{n}} r / R_{\mathrm{n}}, R_{\mathrm{s}} / R_{\mathrm{n}}, k_{\mathrm{s}} / k_{\mathrm{n}}, n, r / L, \mu, \alpha^{t}, \alpha^{r}, V / \sqrt{k_{\mathrm{n}} / \rho}\right\}$. Since the material properties will be studied under quasi-static conditions, the set of parameters can be reduced by removing $V^{\prime} \sqrt{k_{\mathrm{n}} / \rho}, \alpha^{t}$ and $\alpha^{r}$. Further on, assuming that the element size $r$ is small compared to macroscopic dimension $L(r<<$ $L$ ), we can neglect the influence of the parameter $r / L$. The friction coefficient $\mu$ has influence mainly on the post-failure material behaviour, so we can omit it in the relationships for elastic constants and strength parameters. The set of relevant dimensionless parameters is reduced to the following one: $\left\{k_{\mathrm{n}} r / R_{\mathrm{n}}, R_{\mathrm{s}} / R_{\mathrm{n}}, k_{\mathrm{s}} / k_{\mathrm{n}}, n\right\}$. Assuming that the elastic constants are determined in the range in which the failure is not initiated yet, in the relationships for elastic constants we can consider only two dimensionless parameters: $\left\{k_{\mathrm{s}} / k_{\mathrm{n}}, n\right\}$. Finally, the dimensionless functional relationships for macroscopic properties can be assumed in the following form, cf. (Huang 1999): 


$$
\begin{gathered}
E=k_{\mathrm{n}} \Phi_{E}\left(\frac{k_{\mathrm{s}}}{k_{\mathrm{n}}}, n\right) \\
v=\Phi_{v}\left(\frac{k_{\mathrm{s}}}{k_{\mathrm{n}}}, n\right) \\
\sigma_{\mathrm{c}}=\frac{R_{\mathrm{n}}}{r} \Phi_{c}\left(\frac{k_{\mathrm{n}} r}{R_{\mathrm{n}}}, \frac{R_{\mathrm{s}}}{R_{\mathrm{n}}}, \frac{k_{\mathrm{s}}}{k_{\mathrm{n}}}, n\right)
\end{gathered}
$$

The specific form of the dimensionless parameters will be obtained by performing numerical simulations of uniaxial compression test, which will allow us to determine the macroscopic properties for given microscopic parameters.

The set of parameters is not unique and can be modified by taking into account some other parameters that can influence macroscopic properties. In Yang et al. (2006) the minimum and maximum element radii, $r_{\min }$ and $r_{\max }$, respectively, have been included to the relevant parameters, in order to better consider the influence of the element size distribution on macroscopic properties. To some extent, this influence is taken in our formulation by the porosity $n$ which depends on the size distribution, the wider size distribution the lower porosity in the discrete element model can be achieved.

\section{DEM/FEM multiscale model}

\subsection{Main assumptions}

The DEM/FEM multiscale model will be obtained by employing the discrete element and finite element methods in different subdomains of the same body. A special coupling algorithm is used to impose constraints between the subdomains with different scale modelling. The idea of the coupling follows the concept presented by Xiao and Belytschko (2004) for molecular dynamics coupling with a continous model. The DEM and FEM subdomains can overlap each other. In this way a transitory zone between the microscopic-scale model and macroscopic-scale model (finite element) subdomains is introduced. In this zone contributions of each of the two methods to the overall stiffness vary gradually. This allows us to avoid or minimize unrealistic wave reflections at the interface between the DEM and FEM subdomains.

\subsection{Problem formulation}

We consider motion of a deformable body occupying the domain $\Omega$ with the boundary $\Gamma$ (Fig. $7 \mathrm{a}$ ) in the Euclidean space $E^{n_{s d}}$, where $n_{s d}=2$ or 3 . Two different subdomains will be distinguished in the domain $\Omega, \Omega_{F}$ - discretized with finite elements and $\Omega_{D}$ - modelled with discrete elements

$$
\boldsymbol{\Omega}=\boldsymbol{\Omega}_{F} \cup \boldsymbol{\Omega}_{D}
$$

The subdomains $\Omega_{F}$ and $\Omega_{D}$ are not necessarily disjoint - they can overlap each other. The common part of the subdomains $\Omega_{F}$ and $\Omega_{D}$ 


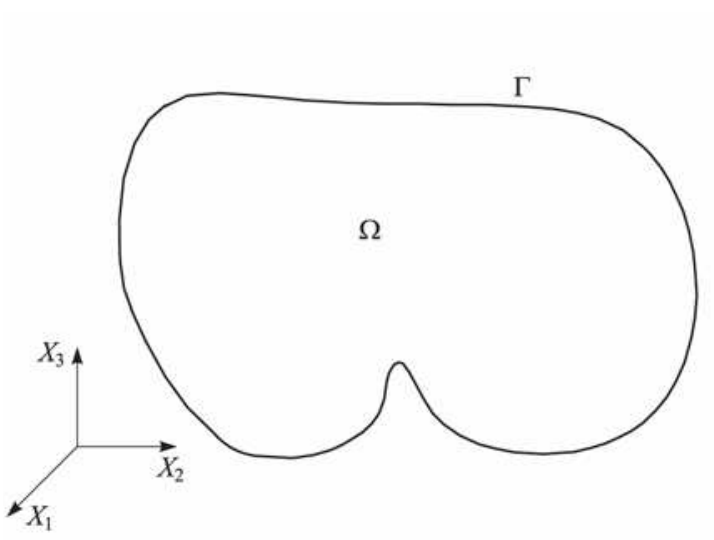

(a)

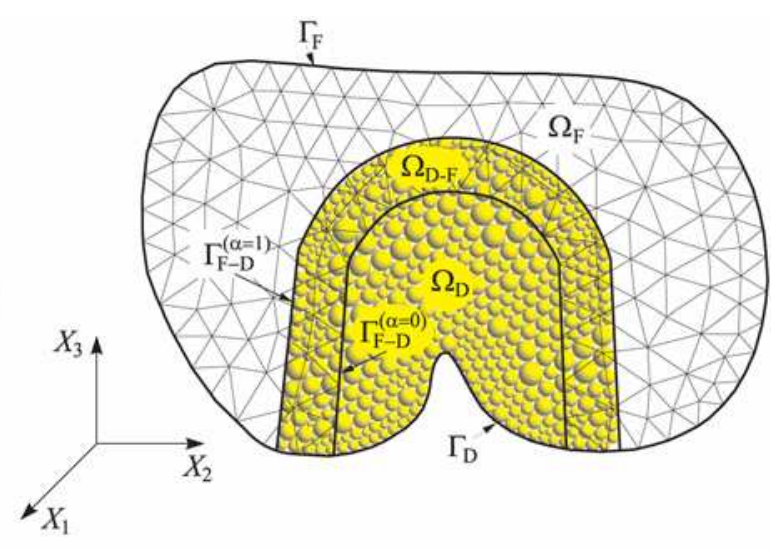

(b)

Fig. 7 The idea of the model: (a) deformable body, (b) multiscale discrete-finite element model

$$
\boldsymbol{\Omega}_{D-F}=\boldsymbol{\Omega}_{F} \cap \boldsymbol{\Omega}_{D} \neq \emptyset
$$

is the part where both discretization types are used. This idea follows that used for molecular dynamics coupling with a continous model in Xiao and Belytschko (2004).

The virtual work in the domain $\mathrm{W}$ will be written as linear combination of the virtual work $\delta W_{F}$ due to the finite element contribution and $\delta W_{D}$ yielded by the discrete element part of the model

$$
\delta W=\alpha \delta W_{F}+(1-\alpha) \delta W_{D}
$$

where the function $\alpha$ is defined in the following way

$$
\alpha(\mathbf{x})= \begin{cases}0 & \text { dla } \mathbf{x} \in \Omega_{D} \backslash \Omega_{D-F} \\ \frac{g(\mathbf{x})}{L(\mathbf{x})} & \text { dla } \mathbf{x} \in \Omega_{D-F} \\ 1 & \text { dla } \mathbf{x} \in \Omega_{F} \backslash \Omega_{D-F}\end{cases}
$$

with $g(\mathbf{x})$ and $L(\mathbf{x})$ being defined in the overlap region $\Omega_{D-F}$.

In the transition zone $\Omega_{D-F}$ the value of function $\alpha$ varies linearly from zero on the surface $\Gamma_{\mathrm{D}-\mathrm{F}}^{(\alpha=0)}$ to unity on the surface $\Gamma_{\mathrm{D}-\mathrm{F}}^{(\alpha=1)}$ (Fig. 8). The surface $\Gamma_{\mathrm{D}-\mathrm{F}}^{(\alpha=0)}$ separates the domain of mixed discrete-continuous modelling from the domain where the discrete element method is used only:

$$
\Gamma_{\mathrm{D}-\mathrm{F}}^{(\alpha=0)}=\Gamma_{\mathrm{F}} \cap \boldsymbol{\Omega}_{\mathrm{D}}
$$

The surface $\Gamma_{\mathrm{D}-\mathrm{F}}^{(\alpha=1)}$, in turn, separates the transition zone from the domain of continuous modelling discretized with finite elements:

$$
\Gamma_{\mathrm{D}-\mathrm{F}}^{(\alpha=1)}=\Gamma_{\mathrm{D}} \cap \boldsymbol{\Omega}_{\mathrm{F}}
$$

Definition of the functions $g(\boldsymbol{x})$ and $L(\boldsymbol{x})$ is shown graphically in Fig. 8. The function $g(\mathbf{x})$ is defined as the shortest distance from the point $\mathbf{x} \in \Omega_{D-F}$ to the boundary of the overlap region $\Gamma_{\mathrm{D}-\mathrm{F}}^{(\alpha) 0)}$ :

$$
g(\boldsymbol{x})=\left\|\overline{\boldsymbol{x}}^{(0)}-\boldsymbol{x}\right\|
$$




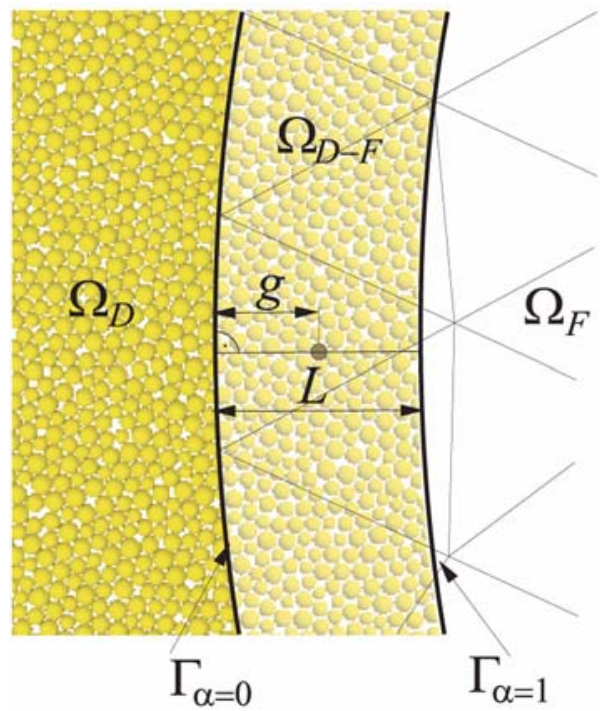

Fig. 8 Geometrical illustration of the parameters defining the scaling function $\alpha$

where

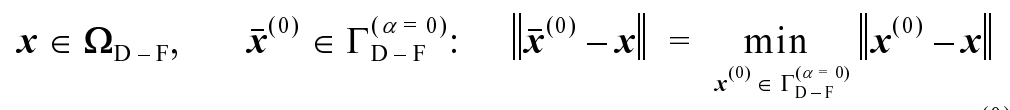

The function $L(\mathbf{x})$ is the width of the overlap zone measured along the vector $\overline{\boldsymbol{x}}^{(0)}-\boldsymbol{x}$.

Finite element discretization employed in the subdomain $\Omega_{\mathrm{F}}$ allows us to express the displacement, velocity and acceleration fields in the discretized region, $\boldsymbol{u}_{\mathrm{F}}, \dot{\boldsymbol{u}}_{\mathrm{F}}$ and $\ddot{\boldsymbol{u}}_{\mathrm{F}}$, respectively,

in terms of shape functions $\mathbf{N}$ and nodal displacements, velocities and accelarations, $\mathbf{r}_{\mathrm{F}}, \dot{\mathbf{r}}_{\mathrm{F}}$ and $\ddot{\mathbf{r}}_{\mathrm{F}}$, respectively, in the following form:

$$
\boldsymbol{u}_{\mathrm{F}}(\boldsymbol{x}, t)=\mathbf{N}(\boldsymbol{x}) \mathbf{r}_{\mathrm{F}}(t), \quad \dot{\boldsymbol{u}}_{\mathrm{F}}(\boldsymbol{x}, t)=\mathbf{N}(\boldsymbol{x}) \dot{\mathbf{r}}_{\mathrm{F}}(t), \quad \ddot{\boldsymbol{u}}_{\mathrm{F}}(\boldsymbol{x}, t)=\mathbf{N}(\boldsymbol{x}) \ddot{\mathbf{r}}_{\mathrm{F}}(t)
$$

In the subdomain $\Omega_{\mathrm{D}}$ the discretemodel is employed. A set of $n_{d e}$ discrete elements $D=\left\{d_{i}\right\}, i=$ $1, n_{d e}$ is introduced. Domain occupied by the discrete elements is a subset of the set $\Omega_{\mathrm{D}}$

$$
\tilde{\Omega}_{\mathrm{D}}=\bigcup_{i=1}^{i=n_{\mathrm{de}}} d_{i} \subset \Omega_{\mathrm{D}}
$$

Configuration and motion of the discrete elements are described by the vectors of translational displacements, $\mathbf{r}_{D}$, translational and rotational velocities, $\dot{\mathbf{r}}_{\mathrm{D}}$ and $\Omega_{\mathrm{D}}$, and translational and rotational accelerations, $\ddot{\mathbf{r}}_{\mathrm{D}}$ and $\dot{\boldsymbol{\Omega}}_{\mathrm{D}}$, respectively.

\subsection{Kinematic constraints}

The subdomains $\Omega_{F}$ and $\Omega_{D}$ are coupled in the common transition zone $\boldsymbol{\Omega}_{\mathrm{D}-\mathrm{F}}$. Coupling is provided by additional kinematic constraints resulting from the assumption that the discrete elements forming the set 


$$
D_{\mathrm{D}-\mathrm{F}}=\left\{d_{i}: \boldsymbol{x}_{d_{i}} \in \boldsymbol{\Omega}_{\mathrm{D}-\mathrm{F}}\right\}
$$

are constrained kinematically by the finite element discretization

$$
\begin{gathered}
\boldsymbol{x}_{d_{i}}=\boldsymbol{x}_{\mathrm{F}}, \quad \forall d_{i} \in \boldsymbol{D}_{\mathrm{D}-\mathrm{F}}, \quad \boldsymbol{x}_{\mathrm{F}} \in \boldsymbol{\Omega}_{\mathrm{D}-\mathrm{F}} \\
\delta \boldsymbol{u}_{d_{i}}=\sum_{j=1}^{n_{w}} N_{j}\left(\boldsymbol{x}_{\mathrm{F}}\right) \delta \boldsymbol{u}_{\mathrm{F} j}, \quad \boldsymbol{v}_{d_{i}}=\sum_{j=1}^{n_{w}} N_{j}\left(\boldsymbol{x}_{\mathrm{F}}\right) \boldsymbol{v}_{\mathrm{F} j}, \quad \dot{\boldsymbol{v}}_{d_{i}}=\sum_{j=1}^{n_{w}} N_{j}\left(\boldsymbol{x}_{\mathrm{F}}\right) \dot{\boldsymbol{v}}_{\mathrm{F} j}
\end{gathered}
$$

Relationships linking virtual displacements, velocities and accelerations of the $i$-th discrete element with respective nodal velocities of the overlapping finite element can be written in the matrix notation as follows:

$$
\delta \boldsymbol{u}_{d_{i}}-\mathbf{N}^{e}\left(\mathbf{x}_{\mathrm{F}}\right) \delta \mathbf{r}_{\mathrm{F}}^{e}=\mathbf{0}, \quad \mathbf{v}_{d_{i}}-\mathbf{N}^{e}\left(\mathbf{x}_{\mathrm{F}}\right) \dot{\mathbf{r}}_{\mathrm{F}}^{e}=\mathbf{0}, \quad \dot{\mathbf{v}}_{d_{i}}-\mathbf{N}^{e}\left(\mathbf{x}_{\mathrm{F}}\right) \delta \ddot{\mathbf{r}}=\mathbf{0}
$$

Splitting the global vectors of displacements, velocities and accelerations $\mathbf{r}_{\mathrm{D}}, \dot{\mathbf{r}}_{\mathrm{D}}$ and $\ddot{\mathbf{r}}_{\mathrm{D}}$, into the unconstrained parts $\mathbf{r}_{\mathrm{DU}}, \dot{\mathbf{r}}_{\mathrm{DU}}$ and $\ddot{\mathbf{r}}_{\mathrm{DU}}$, and constrained ones $\mathbf{r}_{\mathrm{DC}}, \dot{\mathbf{r}}_{\mathrm{DC}} \mathrm{i} \ddot{\mathbf{r}}_{\mathrm{DC}}$, kinematic constraints (73) can be written jointly for all the constrained discrete elements as follows:

$$
\delta \mathbf{r}_{\mathrm{DC}}-\mathbf{N} \delta \mathbf{r}_{\mathrm{F}}=\mathbf{0}, \dot{\mathbf{r}}_{\mathrm{DC}}-\mathbf{N} \dot{\mathbf{r}}_{\mathrm{F}}=\mathbf{0}, \ddot{\mathbf{r}}_{\mathrm{DC}}-\mathbf{N} \ddot{\mathbf{r}}_{\mathrm{F}}=\mathbf{0}
$$

Additional kinematic constraints (74) will be imposed by two alternative methods:

- Lagrange multipliers method,

- penalty function method.

\subsection{Coupled equations of motion - Lagrange multipliers method}

In order to write equations for the coupled system in a compact form, kinematic constraints (74) will be written in a general form:

$$
\chi\left(\delta \mathbf{r}_{\mathrm{F}}, \delta \mathbf{r}_{\mathrm{DC}}\right)=\mathbf{0}
$$

The principle of virtual work for the coupled FEM/DEM system with constraints (75) included by means of the Lagrange multipliers method can be written in the following form:

$$
\begin{gathered}
\delta \mathbf{r}_{\mathrm{F}}^{\mathrm{T}}\left(\overline{\mathbf{M}}_{\mathrm{F}} \ddot{\mathbf{r}}_{\mathrm{F}}+\overline{\mathbf{F}}_{\mathrm{F}}^{\mathrm{int}}-\overline{\mathbf{F}}_{\mathrm{F}}^{\mathrm{ext}}\right)+\delta \mathbf{r}_{\mathrm{DU}}^{\mathrm{T}}\left(\overline{\mathbf{M}}_{\mathrm{DU}} \ddot{\mathbf{r}}_{\mathrm{DU}}-\overline{\mathbf{F}}_{\mathrm{DU}}\right)+\delta \mathbf{r}_{\mathrm{DC}}^{\mathrm{T}}\left(\overline{\mathbf{M}}_{\mathrm{DC}} \ddot{\mathbf{r}}_{\mathrm{DC}}-\overline{\mathbf{F}}_{\mathrm{DC}}\right) \\
+\delta \boldsymbol{\Phi}_{\mathrm{D}}^{\mathrm{T}}\left(\overline{\mathbf{J}}_{\mathrm{D}} \dot{\boldsymbol{\Omega}}_{\mathrm{D}}-\overline{\mathbf{T}}_{\mathrm{D}}\right)+\delta \mathbf{r}_{\mathrm{F}}^{\mathrm{T}}\left[\frac{\partial \chi}{\partial\left(\delta \mathbf{r}_{\mathrm{F}}\right)}\right]^{\mathrm{T}} \lambda+\delta \mathbf{r}_{\mathrm{DC}}^{\mathrm{T}}\left[\frac{\partial \chi}{\partial\left(\delta \mathbf{r}_{\mathrm{DC}}\right)}\right]^{\mathrm{T}} \lambda=0
\end{gathered}
$$

where $\lambda$ is the vector of unknown Lagrange multipliers, $\delta \mathbf{r}_{\mathrm{F}}, \delta \mathbf{r}_{\mathrm{DC}}$ and $\delta \mathbf{r}_{\mathrm{DU}}$ are kinematically admissible virtual displacements, $\delta \Phi_{\mathrm{DU}}$ and $\delta \Phi_{\mathrm{DC}}$-elementary rotations. The global matrices $\overline{\mathbf{M}}_{\mathrm{F}}$, $\overline{\mathbf{M}}_{\mathrm{DU}}, \overline{\mathbf{M}}_{\mathrm{DC}}$ and $\overline{\mathbf{J}}_{\mathrm{D}}$ and vectors $\overline{\mathbf{F}}_{\mathrm{F}}^{\text {int }}, \overline{\mathbf{F}}_{\mathrm{F}}^{\text {ext }}, \overline{\mathbf{F}}_{\mathrm{DU}}, \overline{\mathbf{F}}_{\mathrm{DC}}$ i $\overline{\mathbf{T}}_{\mathrm{D}}$ are assemebled from appropriate elemental matrices and vectors considering contributions from the finite element and discrete element parts according to the assumption expressed by Eq. (62):

$$
\begin{gathered}
\overline{\mathbf{f}}_{e}^{\text {int }}=\int_{\Omega_{e}} \alpha \mathbf{B}^{\mathrm{T}} \sigma \mathrm{d} \Omega_{e} \\
\overline{\mathbf{f}}_{e}^{\text {ext }}=\int_{\Omega_{e}} \alpha \mathbf{N}^{\mathrm{T}} \rho \mathbf{b} \mathrm{d} \Omega_{e}+\int_{\Gamma_{e} \cap \Gamma_{\sigma}} \alpha \mathbf{N}^{\mathrm{T}} \mathbf{t} \mathrm{d} \Gamma_{e}
\end{gathered}
$$




$$
\begin{gathered}
\overline{\mathbf{m}}_{e}=\int_{\Omega_{e}} \alpha \rho \mathbf{N}^{\mathrm{T}} \mathbf{N} \mathrm{d} \Omega_{e} \\
\overline{\mathbf{f}}_{d_{i}}=(1-\alpha) \mathbf{f}_{d_{i}} \\
\overline{\mathbf{m}}_{d_{i}}=(1-\alpha) \mathbf{m}_{d_{i}} \\
\overline{\mathbf{J}}_{d_{i}}=(1-\alpha) \mathbf{J}_{d_{i}} \\
\overline{\mathbf{T}}_{d_{i}}=(1-\alpha) \mathbf{T}_{d_{i}}
\end{gathered}
$$

Taking into account the explicit form of the constraint Eq. (75), Eq. (76) can be written in the following form:

$$
\left\{\begin{array}{c}
\delta \mathbf{r}_{\mathrm{F}} \\
\delta \mathbf{r}_{\mathrm{DU}} \\
\delta \mathbf{r}_{\mathrm{DC}} \\
\delta \boldsymbol{\Phi}_{\mathrm{D}}^{\mathrm{T}}
\end{array}\right\}^{\mathrm{T}}\left(\left[\begin{array}{ccccc}
\overline{\mathbf{M}}_{\mathrm{F}} & \mathbf{0} & \mathbf{0} & \mathbf{0} & -\mathbf{N}^{\mathrm{T}} \\
\mathbf{0} & \overline{\mathbf{M}}_{\mathrm{DU}} & \mathbf{0} & \mathbf{0} & \mathbf{0} \\
\mathbf{0} & \mathbf{0} & \overline{\mathbf{M}}_{\mathrm{DC}} & \mathbf{0} & \mathbf{I} \\
\mathbf{0} & \mathbf{0} & \mathbf{0} & \overline{\mathbf{J}}_{\mathrm{D}} & \mathbf{0}
\end{array}\right]\left\{\begin{array}{l}
\ddot{\mathbf{r}}_{\mathrm{F}} \\
\ddot{\mathbf{r}}_{\mathrm{DU}} \\
\ddot{\mathbf{r}}_{\mathrm{DC}} \\
\dot{\boldsymbol{\Omega}}_{\mathrm{D}} \\
\boldsymbol{\lambda}
\end{array}\right\}-\left\{\begin{array}{l}
\overline{\mathbf{F}}_{\mathrm{F}}^{\mathrm{ext}}-\overline{\mathbf{F}}_{\mathrm{F}}^{\mathrm{int}} \\
\overline{\mathbf{F}}_{\mathrm{DU}} \\
\overline{\mathbf{F}}_{\mathrm{DC}} \\
\overline{\mathbf{T}}_{\mathrm{D}}
\end{array}\right\}\right)=0
$$

Since Eq. (84) must be satisfied for arbitrary admissible variations, the terms in the parenthesis should vanish. Adding the relationship for accelerations $(74)_{3}$ the following equation set is obtained for the coupled system:

$$
\left[\begin{array}{ccccc}
\overline{\mathbf{M}}_{\mathrm{F}} & \mathbf{0} & \mathbf{0} & \mathbf{0} & -\mathbf{N}^{\mathrm{T}} \\
\mathbf{0} & \overline{\mathbf{M}}_{\mathrm{DU}} & \mathbf{0} & \mathbf{0} & \mathbf{0} \\
\mathbf{0} & \mathbf{0} & \overline{\mathbf{M}}_{\mathrm{DC}} & \mathbf{0} & \mathbf{I} \\
\mathbf{0} & \mathbf{0} & \mathbf{0} & \overline{\mathbf{J}}_{\mathrm{D}} & \mathbf{0} \\
-\mathbf{N} & \mathbf{0} & \mathbf{I} & 0 & \mathbf{0}
\end{array}\right]\left\{\begin{array}{l}
\ddot{\mathbf{r}}_{\mathrm{F}} \\
\ddot{\mathbf{r}}_{\mathrm{DU}} \\
\ddot{\mathbf{r}}_{\mathrm{DC}} \\
\dot{\boldsymbol{\Omega}}_{\mathrm{D}} \\
\boldsymbol{\lambda}
\end{array}\right\}=\left\{\begin{array}{l}
\overline{\mathbf{F}}_{\mathrm{F}}^{\mathrm{ext}}-\overline{\mathbf{F}}_{\mathrm{F}}^{\mathrm{int}} \\
\overline{\mathbf{F}}_{\mathrm{DU}} \\
\overline{\mathbf{F}}_{\mathrm{DC}} \\
\overline{\mathbf{T}}_{\mathrm{D}} \\
\mathbf{0}
\end{array}\right\}
$$

with kinematic-type and force-type unknowns. These equations can be solved directly with respect to these unknowns. Another solution scheme has been implemented in our numerical code. It is based on the elimination of Lagrange multipliers $\lambda$ and dependent variables $\mathbf{r}_{\mathrm{DC}}$ before time integration. Performing some algebraic transformations, the reduced equation set is obtained as follows:

$$
\begin{aligned}
\left(\overline{\mathbf{M}}_{\mathrm{F}}+\mathbf{N}^{\mathrm{T}} \overline{\mathbf{M}}_{\mathrm{DC}} \mathbf{N}\right) \ddot{\mathbf{r}}_{\mathrm{F}}=\overline{\mathbf{F}}_{\mathrm{F}}^{\text {ext }}-\overline{\mathbf{F}}_{\mathrm{F}}^{\mathrm{int}}+\mathbf{N}^{\mathrm{T}} \overline{\mathbf{F}}_{\mathrm{DC}} \\
\overline{\mathbf{M}}_{\mathrm{DU}} \ddot{\mathbf{r}}_{\mathrm{DU}}=\overline{\mathbf{F}}_{\mathrm{DU}} \\
\overline{\mathbf{J}}_{\mathrm{D}} \dot{\boldsymbol{\Omega}}_{\mathrm{D}}=\overline{\mathbf{T}}_{\mathrm{D}}
\end{aligned}
$$

These equations can be integrated in time using standard integration schemes used in the explicit finite element and discrete element algorithms without constraints. 


\subsection{Coupled equations of motion - penalty function method}

The principle of virtual work for the coupled DEM-FEMsystem with coupling constraints (75) imposed by the penalty function method can be expressed by the following equation:

$$
\begin{aligned}
& \delta \mathbf{r}_{\mathrm{F}}^{\mathrm{T}}\left(\overline{\mathbf{M}}_{\mathrm{F}} \ddot{\mathbf{r}}_{\mathrm{F}}+\overline{\mathbf{F}}_{\mathrm{F}}^{\mathrm{int}}-\overline{\mathbf{F}}_{\mathrm{F}}^{\mathrm{ext}}\right)+\delta \mathbf{r}_{\mathrm{DU}}^{\mathrm{T}}\left(\overline{\mathbf{M}}_{\mathrm{DU}} \ddot{\mathbf{r}}_{\mathrm{DU}}-\overline{\mathbf{F}}_{\mathrm{DU}}\right)+\delta \mathbf{r}_{\mathrm{DC}}^{\mathrm{T}}\left(\overline{\mathbf{M}}_{\mathrm{DC}} \ddot{\mathbf{r}}_{\mathrm{DC}}-\overline{\mathbf{F}}_{\mathrm{DC}}\right) \\
& +\delta \boldsymbol{\Phi}_{\mathrm{D}}^{\mathrm{T}}\left(\overline{\mathbf{J}}_{\mathrm{D}} \dot{\boldsymbol{\Omega}}_{\mathrm{D}}-\overline{\mathbf{T}}_{\mathrm{D}}\right)+\delta \mathbf{r}_{\mathrm{F}}^{\mathrm{T}}\left[\frac{\partial \chi}{\partial\left(\delta \mathbf{r}_{\mathrm{F}}\right)}\right]^{\mathrm{T}} \chi \mathbf{k}_{\mathrm{DF}}+\delta \mathbf{r}_{\mathrm{DC}}^{\mathrm{T}}\left[\frac{\partial \chi}{\partial\left(\delta \mathbf{r}_{\mathrm{DC}}\right)}\right]^{\mathrm{T}} \chi \mathbf{k}_{\mathrm{DF}}=0
\end{aligned}
$$

where $\mathbf{k}_{\mathrm{DF}}$ is the vector of the discrete penalty function, and global matrices $\overline{\mathbf{M}}_{\mathrm{F}}, \overline{\mathbf{M}}_{\mathrm{DU}}, \overline{\mathbf{M}}_{\mathrm{DC}}$ and $\overline{\mathbf{J}}_{\mathrm{D}}$ global vektors $\overline{\mathbf{F}}_{\mathrm{F}}^{\text {int }}, \overline{\mathbf{F}}_{\mathrm{F}}^{\text {ext }}, \overline{\mathbf{F}}_{\mathrm{DU}}, \overline{\mathbf{F}}_{\mathrm{DC}}$ and $\overline{\mathbf{T}}_{\mathrm{D}}$ are obtained by aggregation of adequate elemental matrices and vectors taking into account appropriate contributions from the discrete and finite element parts to the virtual work in agreement with Eq. (62) according to Eqs. (77), (78), (79), (80) and (81). Taking into account the explicit form of the constraint Eq. (75), Eq. (87) can be written in the following form:

$$
\left\{\begin{array}{c}
\delta \mathbf{r}_{\mathrm{F}} \\
\delta \mathbf{r}_{\mathrm{DU}} \\
\delta \mathbf{r}_{\mathrm{DC}} \\
\delta \boldsymbol{\Phi}_{\mathrm{D}}^{\mathrm{T}}
\end{array}\right\}^{\mathrm{T}}\left(\left[\begin{array}{cccc}
\overline{\mathbf{M}}_{\mathrm{F}} & \mathbf{0} & \mathbf{0} & \mathbf{0} \\
\mathbf{0} & \overline{\mathbf{M}}_{\mathrm{DU}} & \mathbf{0} & \mathbf{0} \\
\mathbf{0} & \mathbf{0} & \overline{\mathbf{M}}_{\mathrm{DC}} & \mathbf{0} \\
\mathbf{0} & \mathbf{0} & \mathbf{0} & \overline{\mathbf{J}}_{\mathrm{D}}
\end{array}\right]\left\{\begin{array}{l}
\ddot{\mathbf{r}}_{\mathrm{F}} \\
\ddot{\mathbf{r}}_{\mathrm{DU}} \\
\ddot{\mathbf{r}}_{\mathrm{DC}} \\
\dot{\boldsymbol{\Omega}}_{\mathrm{D}}
\end{array}\right\}-\left\{\begin{array}{l}
\overline{\mathbf{F}}_{\mathrm{F}}^{\mathrm{ext}}-\overline{\mathbf{F}}_{\mathrm{F}}^{\mathrm{int}}+\mathbf{N}^{\mathrm{T}} \chi \mathbf{k}_{\mathrm{DF}} \\
\overline{\mathbf{F}}_{\mathrm{DU}} \\
\overline{\mathbf{F}}_{\mathrm{DC}}-\chi \mathbf{k}_{\mathrm{DF}} \\
\overline{\mathbf{T}}_{\mathrm{D}}
\end{array}\right\}\right)=0
$$

Since Eq. (88) must be satisfied for arbitrary admissible variations (excluding additional coupling constraints) the terms in the parentheses should vanish, which gives the following equation:

$$
\left[\begin{array}{cccc}
\overline{\mathbf{M}}_{\mathrm{F}} & \mathbf{0} & \mathbf{0} & \mathbf{0} \\
\mathbf{0} & \overline{\mathbf{M}}_{\mathrm{DU}} & \mathbf{0} & \mathbf{0} \\
\mathbf{0} & \mathbf{0} & \overline{\mathbf{M}}_{\mathrm{DC}} & \mathbf{0} \\
\mathbf{0} & \mathbf{0} & \mathbf{0} & \overline{\mathbf{J}}_{\mathrm{D}}
\end{array}\right]\left\{\begin{array}{l}
\ddot{\mathbf{r}}_{\mathrm{F}} \\
\ddot{\mathbf{r}}_{\mathrm{DU}} \\
\ddot{\mathbf{r}}_{\mathrm{DC}} \\
\dot{\boldsymbol{\Omega}}_{\mathrm{D}}
\end{array}\right\}=\left\{\begin{array}{l}
\overline{\mathbf{F}}_{\mathrm{F}}^{\text {ext }}-\overline{\mathbf{F}}_{\mathrm{F}}^{\text {int }}+\mathbf{N}^{\mathrm{T}} \chi \mathbf{k}_{\mathrm{DF}} \\
\overline{\mathbf{F}}_{\mathrm{DU}} \\
\overline{\mathbf{F}}_{\mathrm{DC}}-\chi \mathbf{k}_{\mathrm{DF}} \\
\overline{\mathbf{T}}_{\mathrm{D}}
\end{array}\right\}
$$

Eq. (89) can be integrated in time using the standard explicit schemes used in the finite and discrete element algorithms.

\section{Determination of microscopic parameters}

Determination of rock model parameters is the first step in our simulation of rock cutting process. Values of micromechanical parameters yielding required macroscopic properties of a given rock material will be calculated using the procedure presented in Sec. 4. Macroscopic properties of sandstone have been determined by laboratory tests, unconfined compression test and indirect tension (Brazilian) test, performed in the laboratory of Sandvik Mining and Construction $\mathrm{GmbH}^{3}$ (Zeltweg, Austria). The average parameters from laboratory tests have been taken as the 


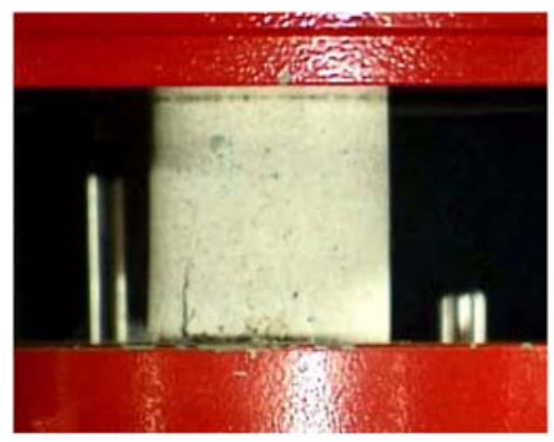

(a)

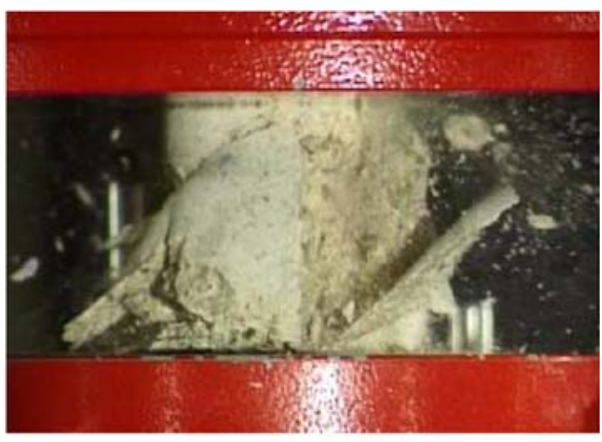

(b)

Fig. 9 Unconfined compression test: (a) sample before the failure, (b) after the failure of the rock sample

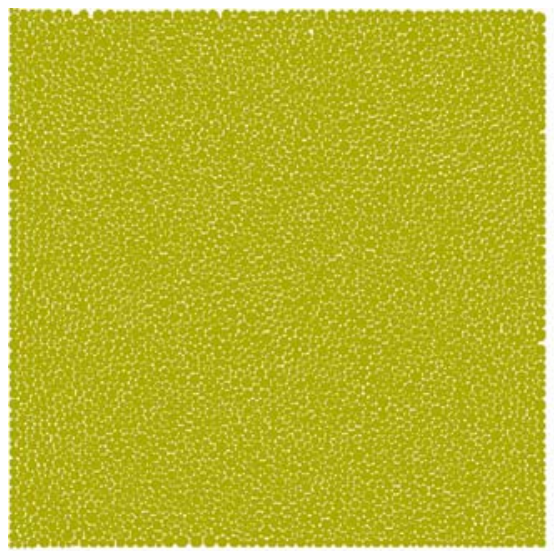

(a)

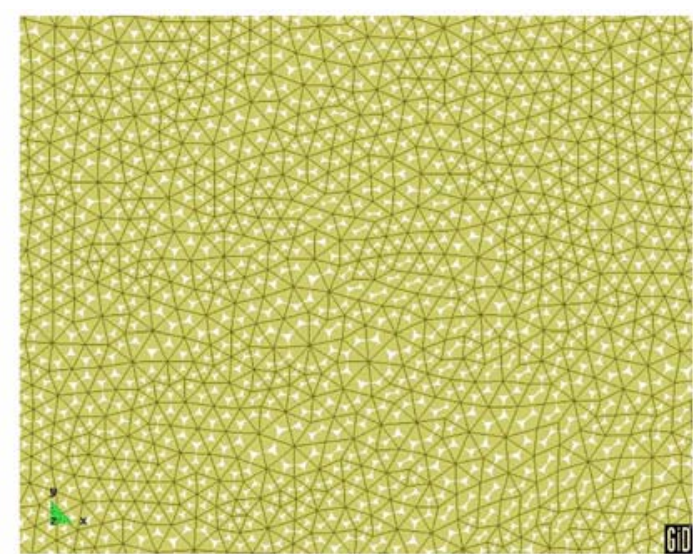

(b)

Fig. 10 Numerical model of unconfined compression test: (a) discrete element model of the rock sample, (b) detail of the model with contact bonds

macroscopic properties of the studied rock as follows: Young modulus $E=18690 \mathrm{MPa}$, Poisson ratio $v=0.18$, unconfined compression strength $\sigma_{c}=127.8 \mathrm{MPa}$, and tensile strength $\sigma_{t}=12.3 \mathrm{MPa}$.

\subsection{Simulation of unconfined compression test}

Fig. 9 shows the unconfined compression test carried out in the laboratory of Sandvik Mining and Construction. Rock samples of equal height and diameter of $50 \mathrm{~mm}$ are used in the testing procedure adopted in this laboratory. Fig. 9 demonstrates failure mode of the rock sample under compressive load.

Numerical simulation of the UCS test will allow us to determine the microscopic constitutive parameters for a material sample modelled with discrete elements. Fig. 10 presents a 2D material sample prepared for numerical testing. The material sample of $50 \times 50 \mathrm{~mm}$ is represented by an assembly of randomly compacted 4979 discs of radii $0.262-0.653 \mathrm{~mm}$. It is shown in Huang (1999) that preparing a well-connected densely packed irregular assembly of particles is the key to successful simulation with discrete elements. Compaction of the particle assembly shown in Fig. 10 is characterized by a porosity of $13 \%$. 


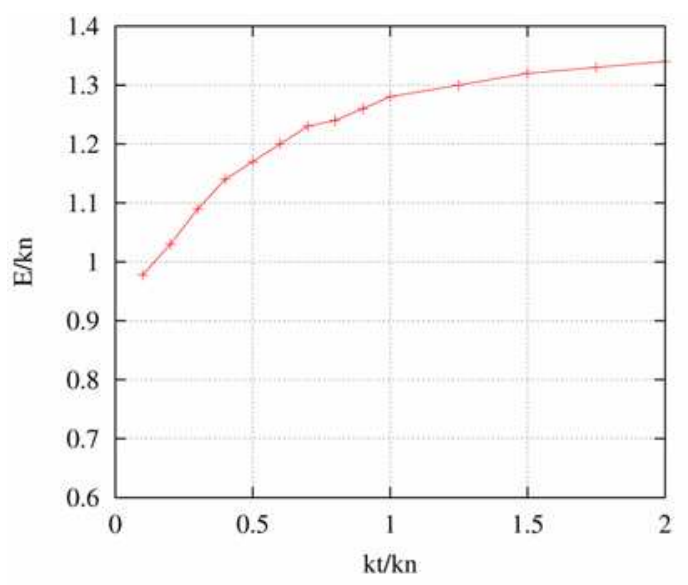

(a)

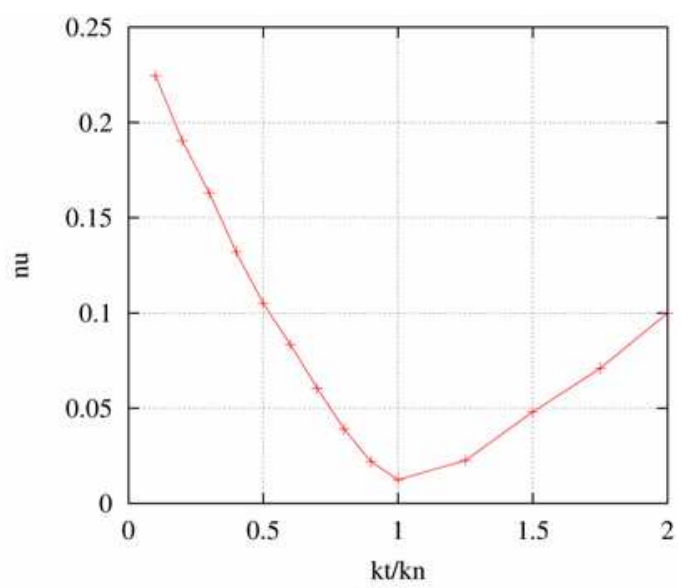

(b)

Fig. 11 Dimensionless relationships between the microscopic parameters and macroscopic elastic constants: (a) relationship for Young's modulus, (b) relationship for Poisson's ratio

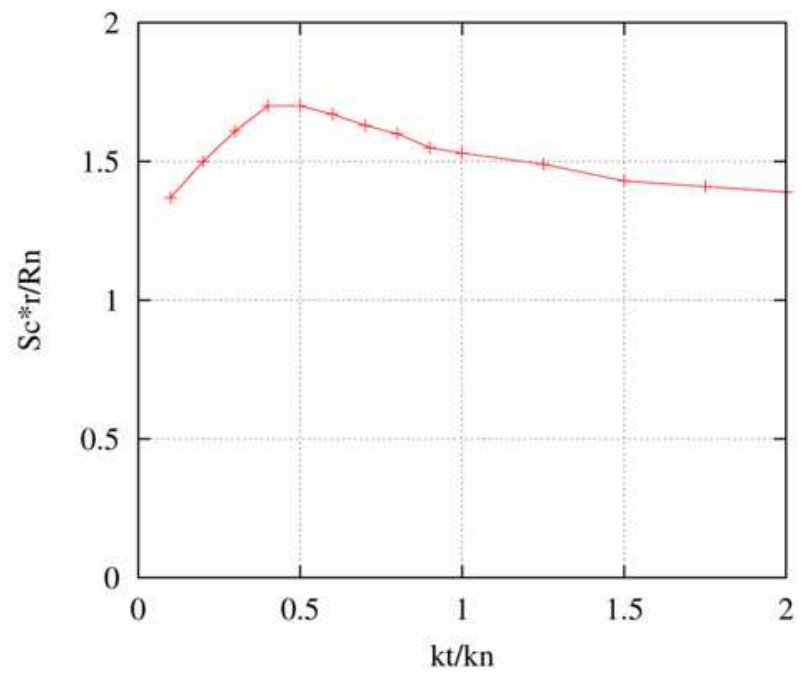

Fig. 12 Dimensionless relationships between the microscopic parameters and compressive strength

The loading has been introduced under kinematic control by prescribing the motion of rigid plates pressing on the top and bottom of the sample. The deformation in the $x$ direction was free. The velocity of the wall displacement was $1 \mathrm{~mm} / \mathrm{s}$ which was found to be sufficiently low to obtain quasi-static loading.

At the beginning a number of simulations have been performed in order to obtain dimensionless relationships between the microscopic parameters and macroscopic properties postulated in Sec. 4.4.2. We have assumed the following set of microscopic parameters $k_{n}=1.5 \cdot 10^{10} \mathrm{~Pa}$, Coulomb friction coefficient $\mu=0.839$ and cohesive bond strengths in the normal and tangential direction, $R_{n}$ $=R_{T}=0.25 \cdot 10^{5} \mathrm{~N} / \mathrm{m}$. Contact stiffness in the tangential direction $k_{s}$ was varying from $1.5 \cdot 10^{9}$ to $3 \cdot 10^{9} \mathrm{~Pa}$. The curves obtained for the dimensionless relationships (57), (58) and (59) are shown in 


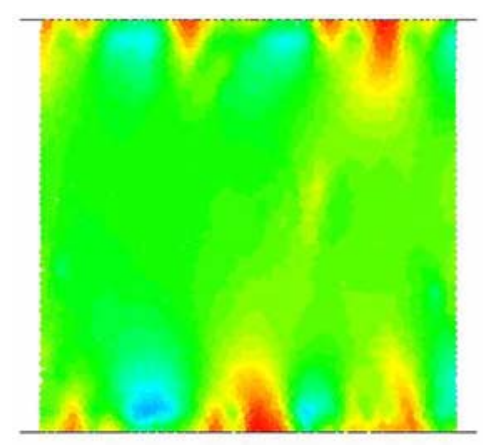

(a) $=0.0014 \mathrm{~s}$

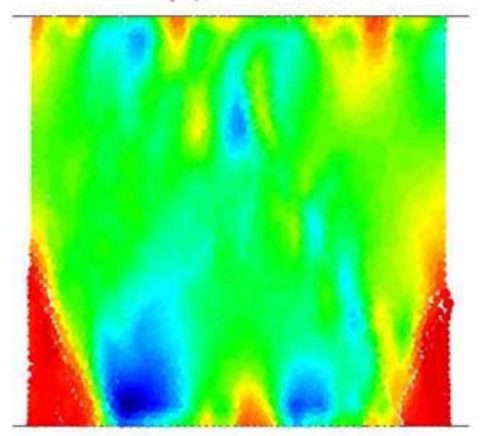

(c) $=0.0018 \mathrm{~s}$

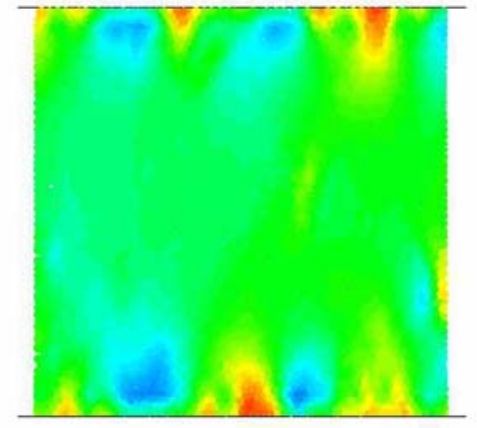

(b) $=0.0016 \mathrm{~s}$

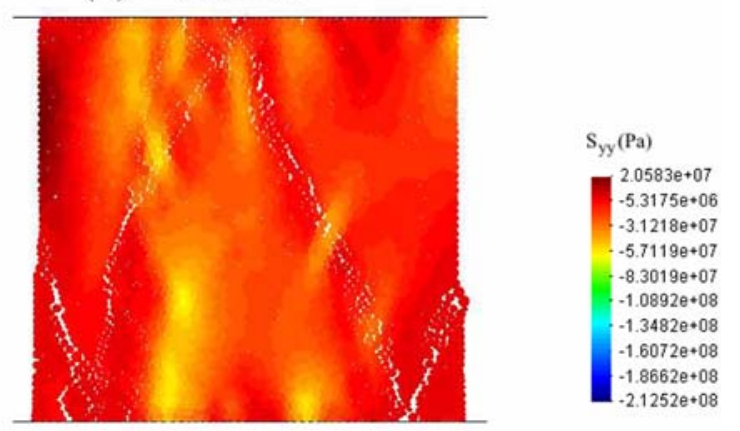

$(\mathrm{d})=0.0020 \mathrm{~s}$

Fig. 13 Simulation of unconfined compression test-failure evolution with distribution of stress along the loading direction

Figs. 11(a), 11(b) and 12, respectively.

Using the dimensionless reltionships the following set of microscopic parameters has been determined for the rock under consideration: $k_{n}=1.61129 \cdot 10^{10} \mathrm{~Pa}$, contact stiffness in the tangential direction $k_{t}=0.3222 \cdot 10^{10} \mathrm{~Pa}$, Coulomb friction coefficient $\mu=0.839$ and cohesive bond strengths in the normal and tangential direction, $R_{n}=R_{t}=0.29 \cdot 10^{5} \mathrm{~N} / \mathrm{m}$.

The failure evolution of the specimen obtained in the simulation with theses parameters is shown in Fig. 13. Comparison of Figs. 13 and 9 shows that numerical analysis yields a failure mode similar to that observed in experiments for brittle rocks. In the presentation of the results in Fig. 13 averaged stresses in the loading direction calculated according to the formulae presented in subsection 4.2 have been plotted. The stresses have been obtained assuming the RVE size of $2.5 r_{\max }, r_{\max }$ is the radius of the largest element in the sample. The stress distributions confirm correct performance of the averaging procedure.

The averaging procedure is also checked in Fig. 14, where two numerical stress-strain curves are plotted, in one of the curves stresses are calculated from the contact forces between the plate and sample, the other one uses the averaged stress in the center of the sample. A good agreement between the two curves can be seen.

The numerical stress-strain curves allow us to evaluate macroscopic properties for the discrete element model. The properties obtained in the numerical simulation of unconfined compression test are as follows: Young modulus $E=18000 \mathrm{MPa}$, Poisson ratio $v=0.20$, uncon - fined compression 


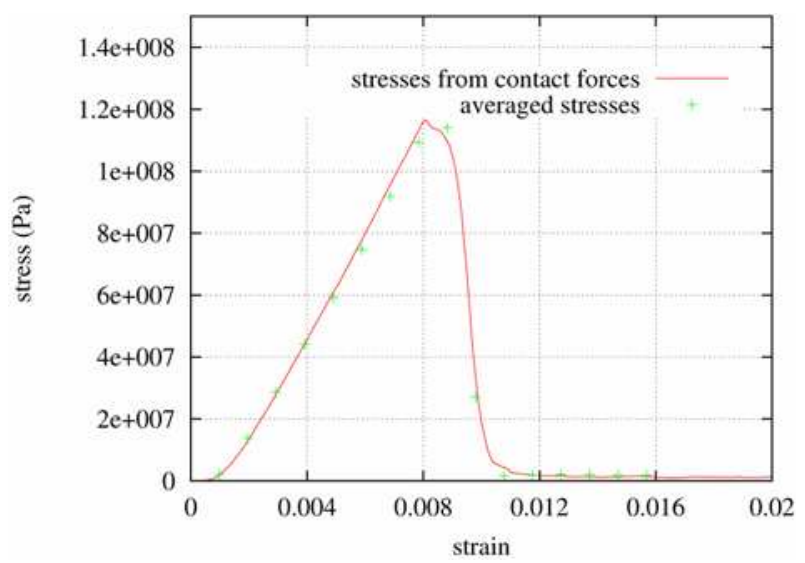

Fig. 14 Simulation of unconfined compression test-stress-strain curve

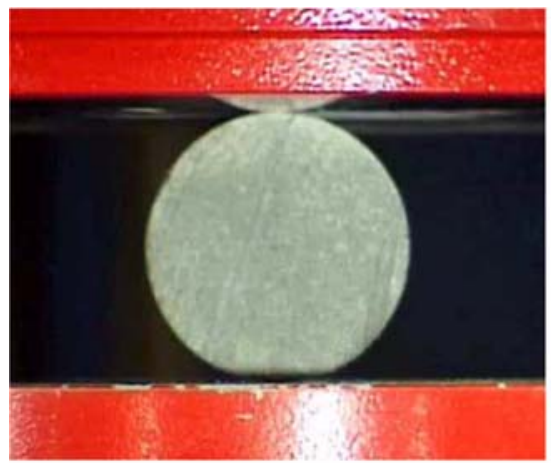

(a)

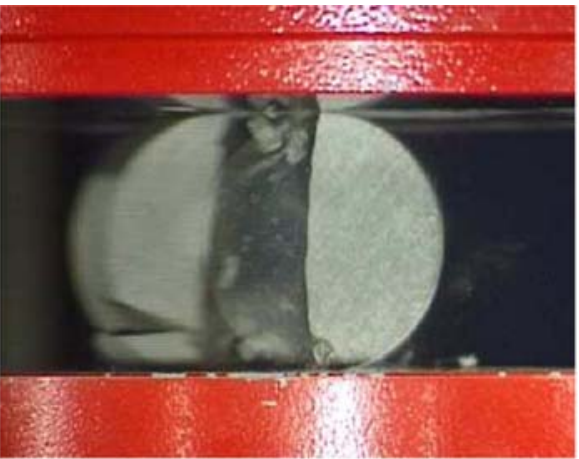

(b)

Fig. 15 Brazilian test: (a) the rock sample before the failure, (b) the rock sample after the failure

strength $\sigma_{c}=116 \mathrm{MPa}$, which we accepted as satisfactorily agreeing with the experimental data.

\subsection{Simulation of indirect tension (Brazilian) test}

Tensile strength of rocks is obtained experimentally from indirect tension (Brasilian) test. In the laboratory of Sandvik Mining and Construction cylindrical samples of diameter of $50 \mathrm{~mm}$ and height (length) of $25 \mathrm{~mm}$ have been used. Laboratory set-up and sample failure are shown in Fig. 15. The failure mode obtained in the analysis is shown in Figs. 16 and 17. Distributions of averaged stresses in the direction parallel and normal to the loading directions shown in Figs. 16 and 17 are in a very good agreement with theoretical solutions (Yua et al. 2006).

The force-displacement relationship obtained in this simulation is plotted in Fig. 18. Taking the maximum force we find the tensile strength as:

$$
\sigma_{t}=\frac{2 P}{\pi L D}=\frac{2 \cdot 1.319 \cdot 10^{6}}{\pi \cdot 1 \cdot 0.05} \mathrm{~Pa}=16.8 \mathrm{MPa}
$$

which we accepted as satisfactorily agreeing with the experimental result, $12.3 \mathrm{MPa}$. 


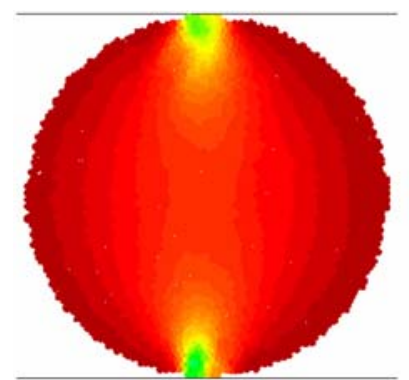

a) $=0.0010 \mathrm{~s}$

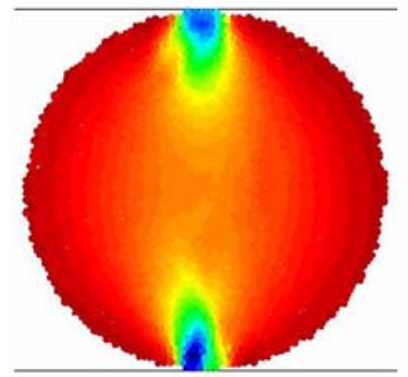

c) $=0.0016 \mathrm{~s}$

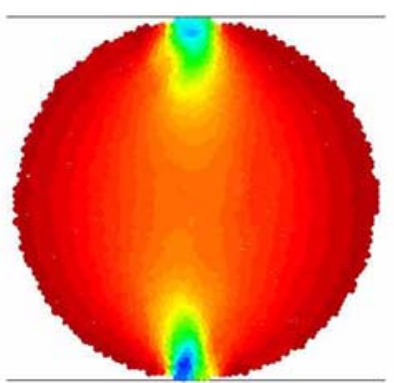

b) $=0.0014 \mathrm{~s}$

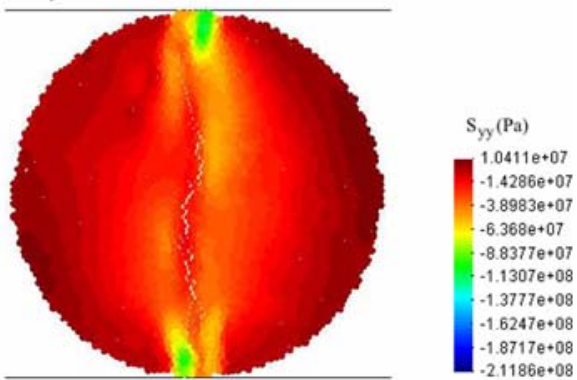

d) $=0.0018 \mathrm{~s}$

Fig. 16 Simulation of Brazilian test-failure of the rock sample with distribution of stress in the direction along the loading

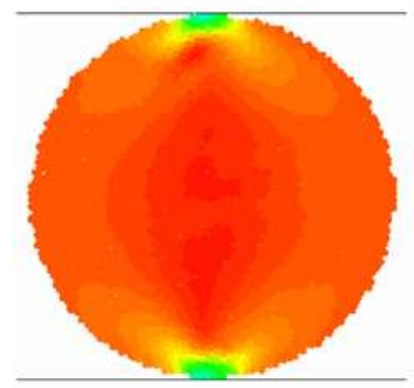

a) $=0.0010 \mathrm{~s}$

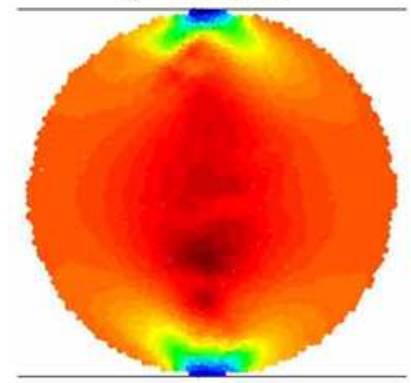

c) $=0.0016 \mathrm{~s}$

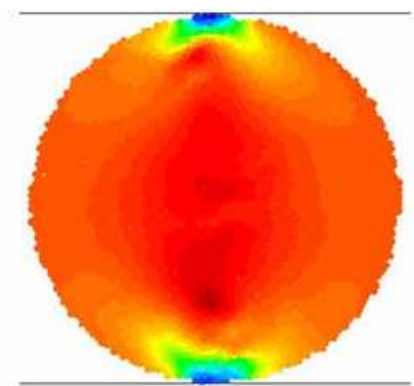

b) $=0.0014 \mathrm{~s}$

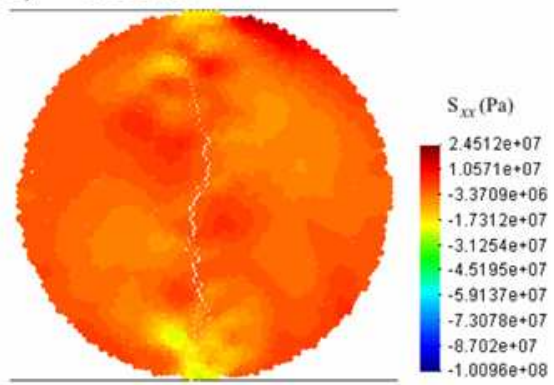

d) $=0.0018 \mathrm{~s}$

Fig. 17 Simulation of Brazilian test-failure of the rock sample with distribution of stress in the direction normal to the loading 


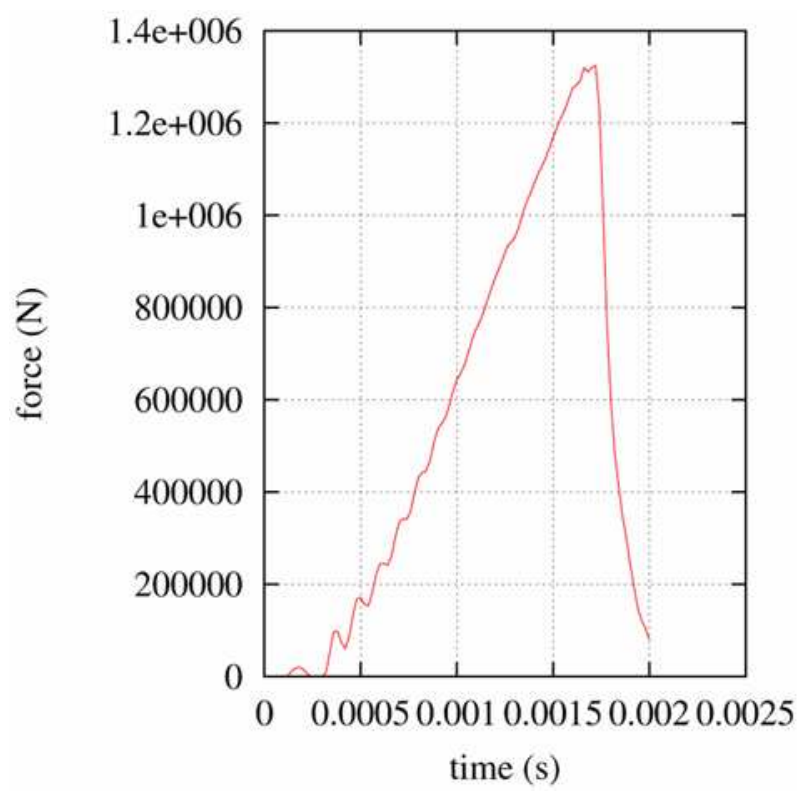

Fig. 18 Simulation of the Brazilian test-stress-displacement curve

\section{Study of wave propagation across the DEM/FEM interface}

Wave propagation is a typical phenomenon observed in the dynamic solution of geomechanical problems modelled with discrete and/or finite element methods. The interface between the FEM and DEM subdomains can introduce an artificial internal boundary causing unrealistic wave reflections. Performance of the coupling method in the presence of wave propagation will be studied in this example.

Wave propagation will be studied under plane strain conditions in the rectangular domain $10 \mathrm{~mm}$ wide and $300 \mathrm{~mm}$ long divided into the DEM and FEM subdomains as it is shown in Fig. 19(a). The longer sides of the rectangle have restrained motion in the transverse direction, the right side $(x=L)$ is fixed and the left side $(x=0)$ is free. Elastic material properties are defined by Young modulus $E=2 \cdot 10^{11} \mathrm{~Pa}$, Poisson's coefficient $v=0.3$ and mass density $\rho=7800 \mathrm{~kg} / \mathrm{m}^{3}$.

The DEM subdomain has been discretized with equal discrete elements $(r=1 \mathrm{~mm})$, and the right subdomain has been modelled with triangular finite elements as shown in Fig. 19(a). Overlapping of the DEM and FEM subdomains is assumed (Fig. 19b). The coupling between the DEM and FEM subdomains has been ensured by the penalty method.

The longitudinal wave pulse has been excited at the left side $(x=0)$ of the rectangle by introducing initial displacements according to the following formula:

$$
u_{x}^{0}=A\left(\cos \frac{2 \pi x}{l}+1\right)
$$

in the region $0 \leq x \leq l / 2$ assuming $A=0.01 \mathrm{~mm}$ i $l=20 \mathrm{~mm}$. The profile of the initial displacements along the $x$-axis is shown in Fig. 19(c). The profile of the wave pulse is shown in Figs. 19(d,e). Wave pulse travels through the DEM subdomain towards the FEM subdomian, passes the DEM/ 


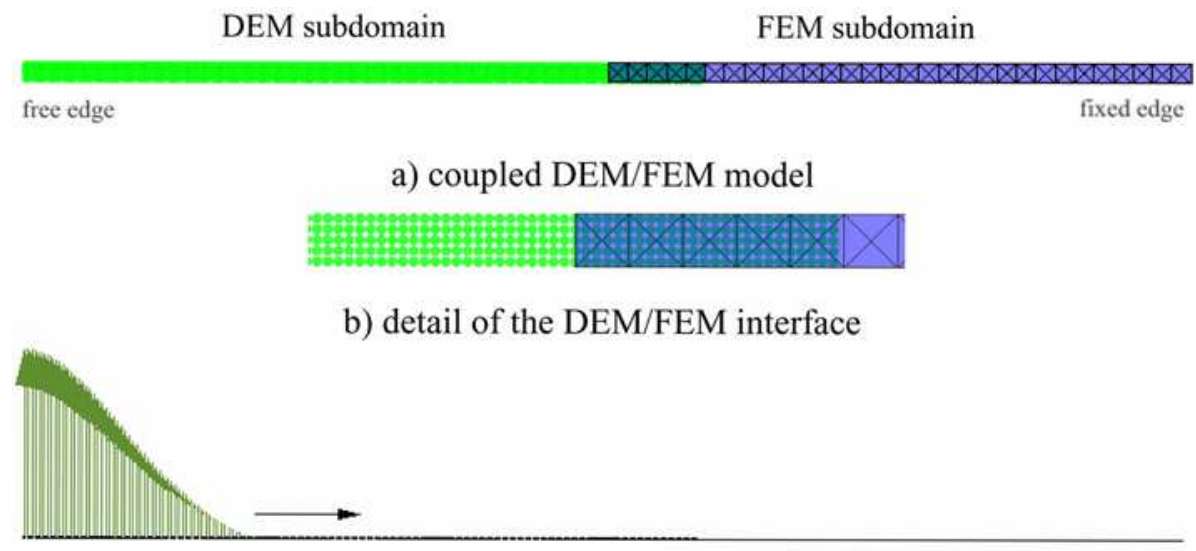

c) wave pulse at $t=0 \mathrm{~s}$

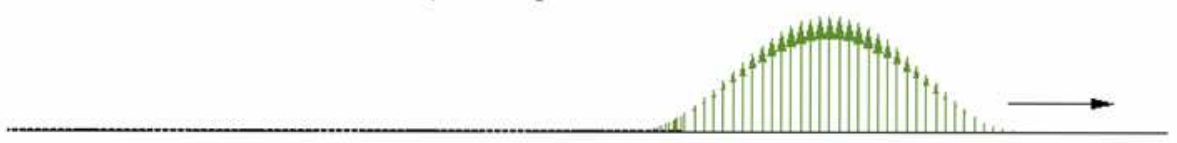

d) wave pulse at $t=8 \cdot 10^{-6} \mathrm{~s}$

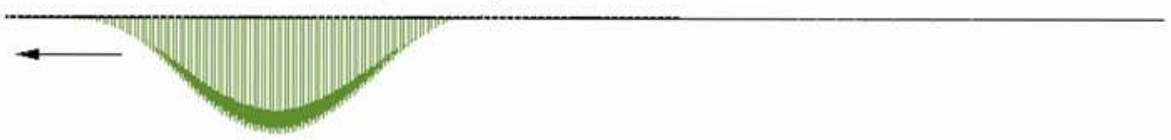

e) wave pulse at $t=2 \cdot 10^{-5} \mathrm{~s}$

Fig. 19 Wave propagation through DEM/FEM model

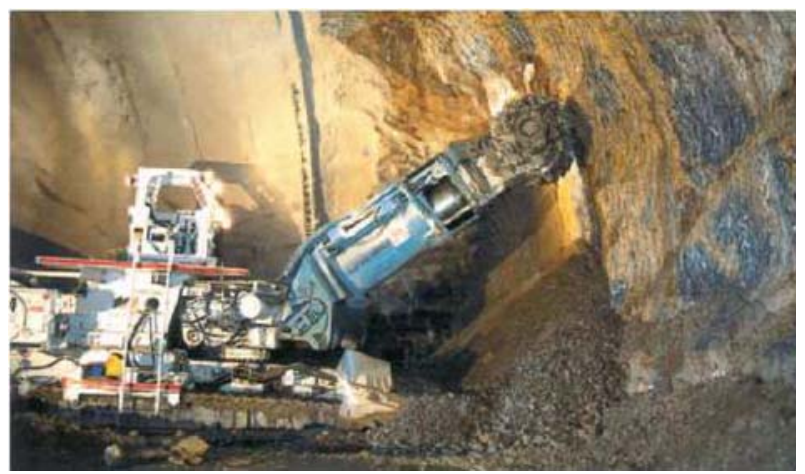

Fig. 20 Excavation with a roadheader

FEM interface without visible reflections, travels through the FEM subdomain towards the clamped side. The pulse reflects from the fixed boundary. The reflected pulse is inverted. It travels back towards the DEM subdomain and passes the DEM/FEM interface in the other direction again without visible reflections. The results demonstrate a correct performance of the coupling algorithm in the presence of wave propagation. 


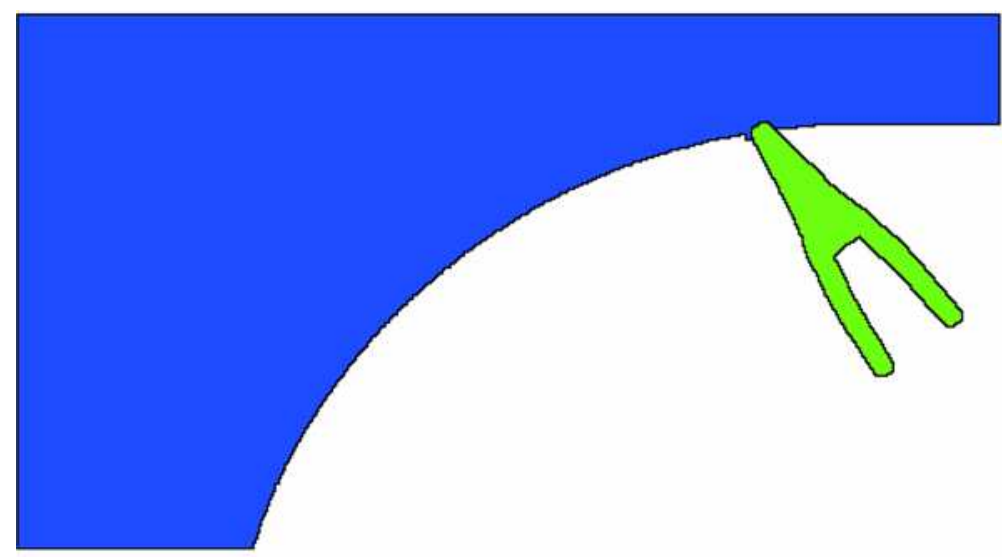

Fig. 21 Model of rock cutting-initial set-up

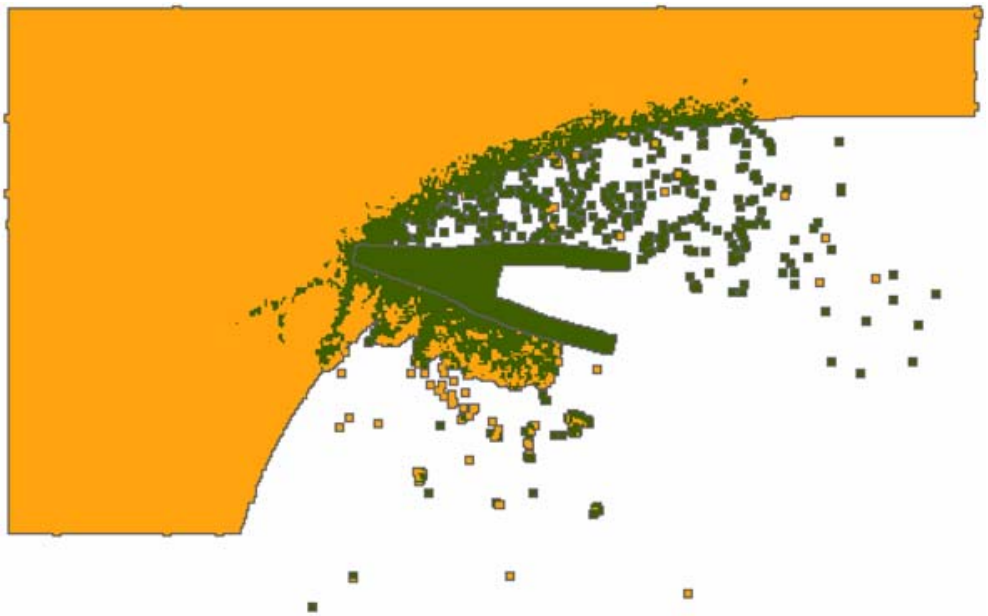

Fig. 22 Simulation of rock cutting by a pick of a road header-failure mode for the discrete element model

\section{Simulation of rock cutting by a pick of a road header using DEM and DEM/FEM models}

Roadheaders are most widely used underground partial-face excavation machines for low to medium strength rocks, in mining engineering as well as in excavation of different underground structures. Fig. 20 shows a roadheader in a real excavation process. In excavation with a roadheader the rock is desintegrated by the action of picks mounted on a rotating cutting head. The cutting head performs also a translational movement controlled by a boom connecting the head to the pedestal of the roadheader.

In this example a process of rock cutting by a single pick of a road header has been analysed using two different models:

- discrete element model 


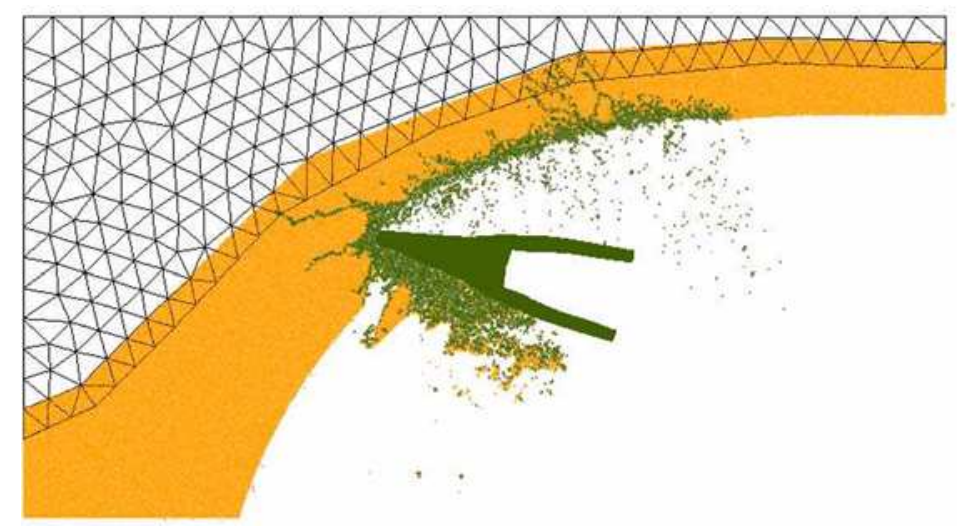

Fig. 23 Simulation of rock cutting by a pick of a road header-failure mode for the hybrid discrete/finite element model

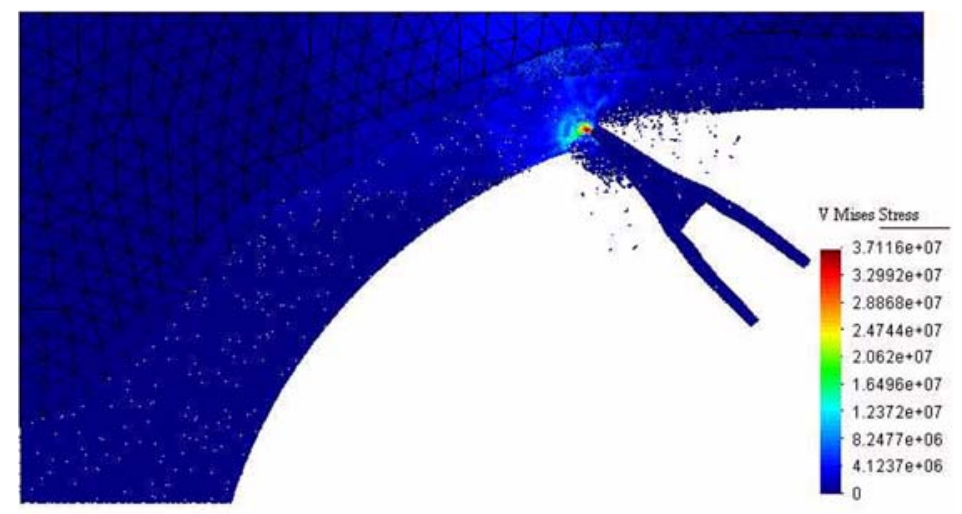

a) $\mathrm{t}=0.28 \mathrm{~s}$

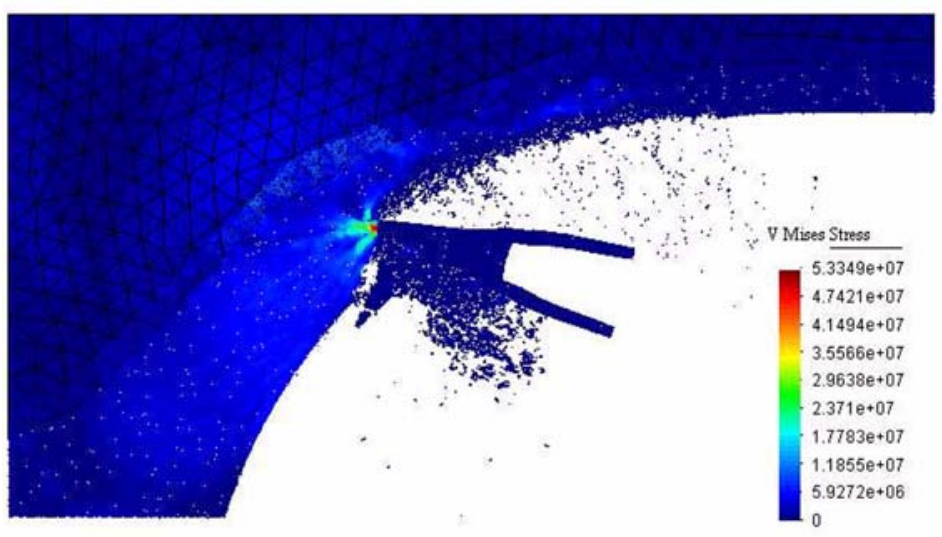

b) $\mathrm{t}=0.84 \mathrm{~s}$

Fig. 24 Simulation of rock cutting by a pick of a road header-equivalent stress distribution in the hybrid discrete/finite element model 
- hybrid discrete/finite element model

\subsection{Discrete element model}

A sample of rock and one pick of a cutter head has been modelled as shown in Fig. 21. Material sample is represented by an assembly of randomly compacted 92000 discs of radii 1-1.5 mm. Model parameters obtained previously for sandstone are assumed for the micromechanical model. The tool is treated as a rigid body. The following parameters have been assumed for the tool-rock interface: contact stiffness modulus $k_{n}=50 \mathrm{GPa}$, Coulomb friction coefficient $\mu=0.4$. The swing velocity of the cutter head was assumed $0.2 \mathrm{~m} / \mathrm{s}$, and rotating velocity $1.6204 \mathrm{~s}^{-1}$, which with the distance of the tooth from the axis of rotation $0.7 \mathrm{~m}$ gives circumferential velocity $1.134 \mathrm{~m} / \mathrm{s}$. Analysis results are shown in Fig. 22. Failure of rock during cutting is shown with damaged zone presented in a different color.

\subsection{Discrete/finite element model}

The model used in the previous section has been modified by replacing discrete elements in a subdomain far away from the fracturing region by finite element discretization. The DEM and FEM subdomains partially overlap. The coupling is enforced by the penalty method.

Results of the numerical analysis for the coupled DEM/FEM model are shown in Figs. 23 and 24. Fig. 23 shows failure mechanism of the rock during cutting.

Fig. 24 shows distribution of equivalent stresses in the rock during cutting. Stresses in the discrete element subdomain are calculated using the averaging algorithm. The tooth is treated as rigid so no stresses are calculated in the tooth (the color in this part has no meaning). Analysis of the stress distribution shows that the stress fields in the two coupled subdomains, calculated in a different way, are consistent. It confirms that coupling algorithm works correctly as well as averaged macroscopic stresses in the discrete element subdomain are calculated correctly.

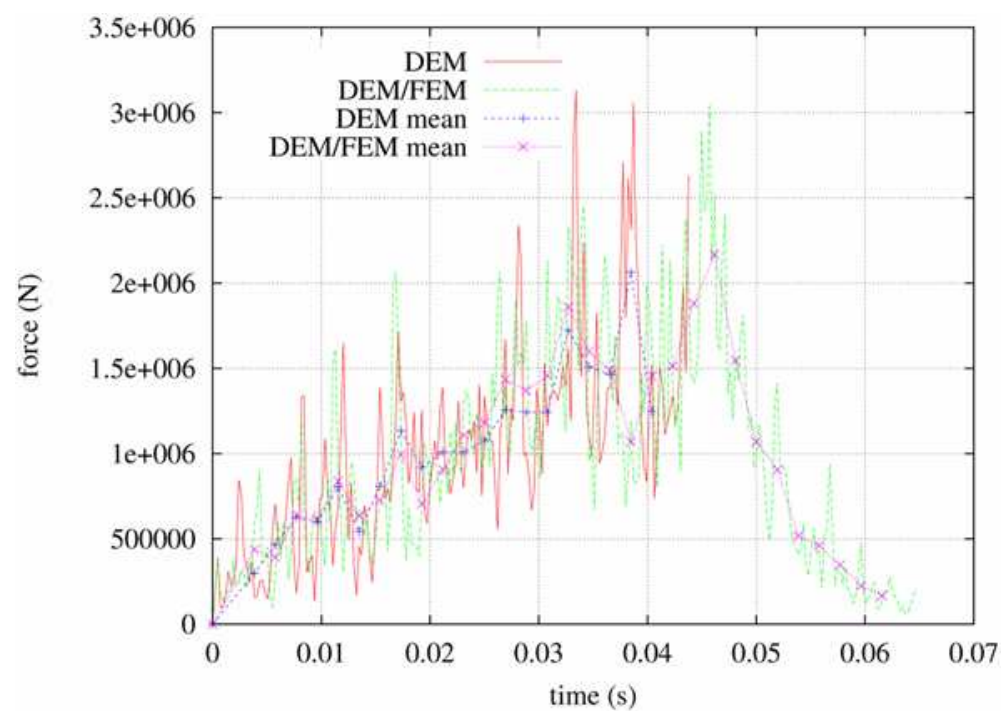

Fig. 25 Cutting force histories for DEM and hybrid DEM/FEM simulations of rock cutting 
Fig. 25 shows the comparison of the cutting forces obtained using the two models, the DEM and DEM/FEM one. Both curves show oscillations typical for cutting of brittle rock. In both cases similar values of amplitudes are observed. Mean values of cutting forces agree very well. This shows that combined DEM/FEM simulation gives similar results to a DEM analysis. This example demonstrates a gain in efficiency when using the DEM/FEM model -computation time has been reduced by half in comparison to the DEM model.

\section{Conclusions}

- Multiscale discrete/finite element modelling can be an optimum modelling method in many problems where local effects require microscopic (discrete) modelling in a limited zone and other parts can be treated as continuous and discretized with finite elements.

- Unified discrete/finite element formulation has been implemented in the authors' own numerical program. The coupling algorithm for overlapping DEM and FEM subdomains has been based on the Lagrange multipliers and penalty methods has been developed.

- Good performance of the coupling algorithm at dynamic problems with wave propagation has been demonstrated

- Microscopic model of rocks based on the discrete element method allows us to represent correctly material failure in laboratory rock tests.

- Multiscale DEM/FEMmodelling has been employed in the analysis of a rock cutting with one pick of a roadheader. Realistic results have been obtained in simulation. Stress fields in the two coupled subdomains, calculated in a different way, are consistent. Averaged macroscopic stresses in the DEM subdomain are calculated correctly.

\section{Acknowledgment}

The work has been partially sponsored by the EU project TUNCONSTRUCT (contract no. IP 011817-2) coordinated by Prof. G. Beer (TU Graz, Austria). The authors thank Egmont Lammer, Hubert Kargl, Uwe Restner and Jan Akermann from Sandvik Mining and Construction in Zeltweg (Austria) for providing experimental results.

\section{References}

Argyris, J. (1982), “An excursion into large rotations”, Comput. Meth. Appl. Mech. Eng., 32, 85-155.

Bagi, K. (1996), "Stress and strain in granular assemblies", Mechanics of Materials, 22, 165-177.

Bardet, J.P. and Proubet, J. (1989) "Application of micromechanics to incrementally nonlinear constitutive equations for granular media", In J. Biarez and R. Gourves, editors, Powders and Grains. Proceedings of the International Conference on Micromechanics of Granular Media, Clermont-Ferrand, 4-8 September 1989, pages 265-270, Rotterdam, Balkema.

Belytschko, T., Smolinski, P. and Liu, W.K. (1985), "Stability of multi-time step partitioned integrators for the first order finite element systems", Comput. Meth. Appl. Mech. Eng., 49, 281-297.

Christensen, R. (1979), Mechanics of Composite Materials. John Wiley, New York.

Cook, B.K. and Jensen, R.P. (2002) editors. "Discrete element methods: numerical modeling of discontinua", 
Proceedings of the Third International Conference on Discrete Element Methods, Santa Fe, New Mexico.

Cundall, P.A. and Strack, O.D.L. (1979), “A discrete numerical method for granular assemblies", Geotechnique, 29, 47-65.

Cundall, P.A. (1988), "Formulation of a three dimensional distinct element model-Part I. A scheme to detect and represent contacts in a system of many polyhedral blocks", Int. J. Rock Mech., Min. Sci. \& Geomech. Abstr., 25(3), 107-116.

Huang, H. (1999), "Discrete element modeling of tool-rock interaction”, PhD thesis, University of Minnesota.

Kouznetsova, V. (2002), "Computational homogenization for the multi-scale analysis of multiphase materials", $\mathrm{PhD}$ thesis, Technische Universiteit of Eindhoven.

Langhaar, H.L. (1951) Dimensional Analysis and Theory of Models. Wiley.

Lätzel, M., Luding, S. and Herrmann, H.J. (2000), "Macroscopic material properties from quasistatic, microscopic simulations of a two-dimensional shear-cell", Granular Matter, 2, 123-135.

Lätzel, M. (2003), "From microscopic simulations towards a macroscopic description of granular media", $\mathrm{PhD}$ thesis, University of Stuttgart.

Liao, C.L. and Chan, T.C. (1997), "Generalized constitutive relation for a randomly packed particle assembly", Computers and Geotechnics, 20, 345-363.

Liao, Ch.-L., Chang, T.-P., Young, D.-H. and Chang, Ch.S. (1997), "Stress-strain relationship for granular materials based on the hypothesis of best fit", Int. J. Solids and Structures, 34, 4087-4100.

Luding, S. (2004), "Micro-macro transition for anisotropic, aperiodic, granular materials", Int. J. Solids and Structures, 41, 5821-5836.

Miehe, C. (1996), "Numerical computation of algorithmic (consistent) tangent moduli in largestrain computational elasticity", Comput. Meth. Appl. Mech. Eng., 134, 223-240.

Miehe, C., Schröder, J. and Becker, M. (2002), "Computational homogenization analysis in finite elasticity: Material and structural instabilities on the micro- and macro-scales of periodic composites and their interaction", Comput. Meth. Appl. Mech. Eng., 191, 4971-5005.

Munjiza, A. (2004) The Combined Finite-Discrete Element Method. Wiley.

Nemat-Nasser, S. and Hori, M. (1993), Micromechanics: overall properties of heterogeneous materials. North Holland, Amsterdam.

Oñate, E. and Rojek, J. (2004), "Combination of discrete element and finite element methods for dynamic analysis of geomechanics problems", Comput.Meth. Appl.Mech. Eng., 193, 3087-3128.

Potyondy, D.O. and Cundall, P.A. (2004), "A bonded-particle model for rock", Int. J. Rock Mech. Min. Sci., 41, $1329-1364$.

Ramm, E., D'Addetta, G.A. and Leukart, M. (2003), "Interrelations between continuum and discontinuum models for geomaterials", VII International Conference on Computational Plasticity COMPLAS 2003, Barcelona.

Rojek, J. and Oñate, E. (2004), "Unified DEM/FEM approach to geomechanics problems", Proceedings of ComputationalMechanics WCCM VI in conjunction with APCOM04, Beijing, China, Sept. 5-10.

Rojek, J. (2007), "Modelling and simulation of complex problems of nonlinear mechanics using the finite and discrete element methods" (in Polish). Habilitiation Thesis, Institute of Fundamental Technological Research Polish Academy of Sciences, Warsaw.

Rojek, J., Oñate, E., Zarate, F. and Miquel, J. (2001), "Modelling of rock, soil and granular materials using spherical elements", 2nd European Conference on Computational Mechanics ECCM-2001, Cracow, 26-29 June.

Xiao, S.P. and Belytschko, T. (2004), "A bridging domain method for coupling continua with molecular dynamics", Comput. Meth. Appl. Mech. Eng., 193, 1645-1669.

Yang, B., Jiao, Y. and Lei, S. (2006), "A study on the effects of microparameters on macroproperties for specimens created by bonded particles", Eng. Comput., 23(6), 607-631.

Young, R.P., Collins, D.S., Hazzard, J., Heath, A., Pettitt, W.S., Baker, C., Billaux, D., Cundall, P., Potyondy, D., Dedecker, F., Svemar, C. and Lebon, P. (2004), "An innovative 3-D numerical modelling procedure for simulating repository-scale excavations in rock-SAFETI", Proceedings of the Euradwaste04 Conference on Radioactive Waste Management Community Policy and Research Initiatives, Luxembourg.

Yua, Y., Yinb, J. and Zhong, Z. (2006), "Shape effects in the Brazilian tensile strength test and a 3D FEM correction", Int. J. Rock Mech. Min. Sci., 43, 623-627. 